\title{
MULTIPLICATION OPERATORS ON THE BERGMAN SPACE VIA THE HARDY SPACE OF THE BIDISK
}

\author{
KUNYU GUO, SHUNHUA SUN, DECHAO ZHENG AND CHANGYONG ZHONG
}

\begin{abstract}
In this paper, we develop a machinery to study multiplication operators on the Bergman space via the Hardy space of the bidisk. Using the machinery we study the structure of reducing subspaces of a multiplication operator on the Bergman space. As a consequence, we completely classify reducing subspaces of the multiplication operator by a Blaschke product $\phi$ with order three on the Bergman space to solve a conjecture of Zhu [40].
\end{abstract}

\section{INTRODUCTION}

Let $\mathbb{D}$ be the open unit disk in $\mathbb{C}$. Let $d A$ denote the Lebesgue area measure on the unit disk $\mathbb{D}$, normalized so that the measure of $\mathbb{D}$ equals 1 . The Bergman space $L_{a}^{2}$ is the Hilbert space consisting of the analytic functions on $\mathbb{D}$ that are also in the space $L^{2}(\mathbb{D}, d A)$ of square integrable functions on $\mathbb{D}$.

Our main objective is to study multiplication operators on $L_{a}^{2}$ by bounded analytic functions on the unit disk $\mathbb{D}$ via the Hardy space of bidisk. The theme is to use the theory of multivariable operators to study a single operator. Our main idea is to lift the Bergman shift up as the compression of a commuting pair of isometries on a nice subspace of the Hardy space of bidisk. This idea was used in studying the Hilbert modules by R. Douglas and V. Paulsen [12], operator theory in the Hardy space over the bidisk by R. Dougals and R. Yang [13], [37], [38] and [39]; the higher-order Hankel forms by S. Ferguson and R. Rochberg [10] and [11] and the lattice of the invariant subspaces of the Bergman shift by S. Richter [22].

For a bounded analytic function $\phi$ on the unit disk, the multiplication operator $M_{\phi}$ is defined on the Bergman space $L_{a}^{2}$ given by $M_{\phi} h=\phi h$ for $h \in L_{a}^{2}$. Let $e_{n}=\sqrt{n+1} z^{n}$. Then $\left\{e_{n}\right\}_{0}^{\infty}$ form an orthonormal basis of the Bergman space $L_{a}^{2}$. On the basis $\left\{e_{n}\right\}$, the multiplication operator $M_{z}$ by $z$ is a weighted shift operator, called the Bergman shift: $M_{z} e_{n}=\sqrt{\frac{n+1}{n+2}} e_{n+1}$.

The multiplication operators on the Bergman space possess a very rich structure theory. Even the lattice of the invariant subspaces of the Bergman shift $M_{z}$ is huge [4]. The Bergman shift $M_{z}$ has a universal property [4]: for any strict contraction $S$ on a Hilbert space $H$, there always exist a pair of invariant subspaces of $M_{z}$, say $\mathcal{M}$ and $\mathcal{N}$ in Lat $M_{z}$ ( the invariant subspace lattice of $M_{z}$ is the set of subspaces $\mathcal{M}$ of $L_{a}^{2}$ with $\left.M_{z} \mathcal{M} \subset \mathcal{M}\right)$, such that $S \cong P_{\mathcal{M} \odot \mathcal{N}}\left\{\left.M_{z}\right|_{\mathcal{M} \ominus \mathcal{N}}\right\}$, where $P_{\mathcal{M} \ominus \mathcal{N}}$ denotes the orthogonal projection of $L_{a}^{2}(\mathbb{D})$ onto $\mathcal{M} \ominus \mathcal{N}$. This indicates that existence of the invariant subspace problem for Hilbert space operator is equivalent to whether $\operatorname{Lat}_{z}$ is saturated, i.e., for any $\mathcal{M}, \mathcal{N} \in \operatorname{Lat} M_{z}$, with $\mathcal{M} \supset \mathcal{N}$ and $\operatorname{dim}(\mathcal{M} \ominus \mathcal{N})=\infty$, whether there always exists some $\Omega \in \operatorname{Lat} M_{z}$ such that

$$
\mathcal{N} \varsubsetneqq \Omega \varsubsetneqq \mathcal{M} \text {. }
$$

Let $\mathbb{T}$ denote the unit circle. The torus $\mathbb{T}^{2}$ is the Cartesian product $\mathbb{T} \times \mathbb{T}$. The Hardy space $H^{2}\left(\mathbb{T}^{2}\right)$ over the bidisk is $H^{2}(\mathbb{T}) \otimes H^{2}(\mathbb{T})$. Let $P$ be the orthogonal projection from the space $L^{2}\left(\mathbb{T}^{2}\right)$ of the Lebesgue square integrable functions on the torus $\mathbb{T}^{2}$ onto $H^{2}\left(\mathbb{T}^{2}\right)$. The Toeplitz operator on $H^{2}\left(\mathbb{T}^{2}\right)$ with symbol $f$ in $L^{\infty}\left(\mathbb{T}^{2}\right)$ is defined by $T_{f}(h)=P(f h)$, for $h \in H^{2}\left(\mathbb{T}^{2}\right)$.

The first author and the second author were supported in part by the National Natural Science Foundation of China. The third author was partially supported by the National Science Foundation. 
Clearly, $T_{z}$ and $T_{w}$ are a pair of doubly commuting pure isometries on $H^{2}\left(\mathbb{T}^{2}\right)$. For each integer $n \geq 0$, let

$$
p_{n}(z, w)=\sum_{i=0}^{n} z^{i} w^{n-i}=\frac{z^{n+1}-w^{n+1}}{z-w} .
$$

Let $\mathcal{H}$ be the subspace of $H^{2}\left(\mathbb{T}^{2}\right)$ spanned by functions $\left\{p_{n}\right\}_{n=0}^{\infty}$. The orthogonal complement of $\mathcal{H}$ in $H^{2}\left(\mathbb{T}^{2}\right)$ is

$$
[z-w]=\text { closure }_{H^{2}\left(\mathbb{T}^{2}\right)}\left\{(z-w) H^{2}\left(\mathbb{T}^{2}\right)\right\} .
$$

Then $[z-w]$ is an invariant subspace of analytic Toeplitz operators $T_{f}$ for $f \in H^{\infty}\left(\mathbb{T}^{2}\right)$. Let $P_{\mathcal{H}}$ be the orthogonal projection from $L^{2}\left(\mathbb{T}^{2}\right)$ onto $\mathcal{H}$. It is easy to check that

$$
\left.P_{\mathcal{H}^{T}}\right|_{\mathcal{H}}=\left.P_{\mathcal{H}} T_{w}\right|_{\mathcal{H}}
$$

Let $\mathcal{B}$ denote the operator above. It was shown explicitly in [29] and implicitly in [12] that $\mathcal{B}$ is unitarily equivalent to the Bergman shift $M_{z}$ on the Bergman space $L_{a}^{2}$ via the following unitary operator $U: L_{a}^{2}(\mathbb{D}) \rightarrow \mathcal{H}$

$$
U z^{n}=\frac{p_{n}(z, w)}{n+1}
$$

Clearly, for each $f(z, w) \in \mathcal{H}$,

$$
\left(U^{*} f\right)(z)=f(z, z),
$$

for $z \in D$. The simple observation that $p_{n}(z, w)=\frac{z^{n+1}-w^{n+1}}{z-w}$ gives that for each $f(z, w) \in \mathcal{H}$, there is a function $\tilde{f}(z)$ in the Dirichlet space $\mathcal{D}$ such that

$$
f(z, w)=\frac{\tilde{f}(z)-\tilde{f}(w)}{z-w} .
$$

Thus, for each Blaschke product $\phi(z)$ with finite order, the multiplication operator $M_{\phi}$ on the Bergman space is unitarily equivalent to $\phi(\mathcal{B})$ on $\mathcal{H}$.

In this paper we will study the operator $\phi(\mathcal{B})$ on the Hardy space of the bidisk to shed light on properties of the multiplication operator $M_{\phi}$. This method seems to be effective in dealing with the multiplication operators on the Bergman space because functions in the Hardy space of the bidisk behave slightly better than the functions in the Bergman space.

The difficulty to study $\mathcal{B}$ on $\mathcal{H}$ is to get better understanding the projection $P_{\mathcal{H}}$. The price that we pay is that we will get a lot of mileage from developing a "heavy" machinery on the Hardy space of the bidisk how to get rid of $P_{\mathcal{H}}$ in the expression

$$
\phi(\mathcal{B})^{n} f=\frac{1}{n+1} P_{\mathcal{H}}\left(p_{n}(\phi(z), \phi(w)) f\right),
$$

for $f \in \mathcal{H}$. To do this, letting $L_{0}$ denote the space $\operatorname{ker} T_{\phi(z)}^{*} \cap \operatorname{ker} T_{\phi(w)}^{*} \cap \mathcal{H}$, for each $e \in L_{0}$, we construct functions $\left\{d_{e}^{k}\right\}$ in Section 3.1 and $d_{e}^{0}$ in Section 3.2 such that for each $l \geq 1$,

$$
p_{l}(\phi(z), \phi(w)) e+\sum_{k=0}^{l-1} p_{k}(\phi(z), \phi(w)) d_{e}^{l-k} \in \mathcal{H}
$$

and

$$
p_{l}(\phi(z), \phi(w)) e+p_{l-1}(\phi(z), \phi(w)) d_{e}^{0} \in \mathcal{H} .
$$

On one hand, we have a precise formula of $d_{e}^{0}$. On the other hand, $d_{e}^{k}$ is orthogonal to $L_{0}$. These constructions are useful in studying the reducing subspaces of $\phi(\mathcal{B})$. A reducing subspace $\mathcal{M}$ for an operator $T$ on a Hilbert space $H$ is a subspace $\mathcal{M}$ of $H$ such that $T \mathcal{M} \subset \mathcal{M}$ and $T^{*} \mathcal{M} \subset \mathcal{M}$. A reducing subspace $\mathcal{M}$ of $T$ is called minimal if only reducing subspaces contained in $\mathcal{M}$ are $\mathcal{M}$ and $\{0\}$. As in [16], a subspace $\mathcal{N}$ of $H$ is a wandering subspace of $T$ if $\mathcal{N}$ is orthogonal to $T^{n} \mathcal{N}$ for each $n=1,2, \cdots$. If $\mathcal{M}$ is an invariant subspace of $T$, it is clear that $\mathcal{M} \ominus T \mathcal{M}$ is a wandering subspace of $T$, and we will refer this subspace as the wandering subspace of $\mathcal{M}$. 
In fact, for a reducing subspace $\mathcal{M}$ of $\phi(\mathcal{B})$, and $e$ in the wandering subspace of $\mathcal{M}$

$$
p_{l}(\phi(z), \phi(w)) e+\sum_{k=0}^{l-1} p_{k}(\phi(z), \phi(w)) d_{e}^{l-k} \in \mathcal{M} .
$$

Although for a Blaschke product $\phi$ of finite order, $M_{\phi}$ is not an isometry, using the machinery on the Hardy space of bidisk we will show that there exists a unique reducing subspace $\boldsymbol{\mathcal { M }}_{0}$, the so called distinguished subspace, on which the restriction of $M_{\phi}$ is unitarily equivalent to the Bergman shift, which will play an important role in classifying reducing subspaces of $M_{\phi}$. The functions $d_{e}^{1}$ and $d_{e}^{0}$ have the following relation.

Theorem 1. If $\mathcal{M}$ is a reducing subspace of $\phi(\mathcal{B})$ orthogonal to the distinguished reducing subspace $\mathcal{M}_{0}$, for each $e$ in the wandering subspace for $\mathcal{M}$, then there is an element $\tilde{e}$ in the wandering subspace for $\mathcal{M}$ and a number $\lambda$ such that

$$
d_{e}^{1}=d_{e}^{0}+\tilde{e}+\lambda e_{0} .
$$

To understand the structure of minimal reducing subspaces of $\phi(\mathcal{B})$ we lift a reducing subspace of $\phi(\mathcal{B})$ as a reducing subspace of the pair of doubly commuting isometries $T_{\phi(z)}$ and $T_{\phi(w)}$. For a given reducing subspace $\mathcal{M}$ of $\phi(\mathcal{B})$, define the lifting $\widetilde{\mathcal{M}}$ of $\mathcal{M}$

$$
\widetilde{\mathcal{M}}=\operatorname{span}\left\{\phi(z)^{l} \phi(w)^{k} \mathcal{M}, l, k \geq 0\right\} .
$$

Since $\mathcal{M}$ is a reducing subspace of $\phi(\mathcal{B})$ and $\widetilde{\mathcal{M}}$ is a reducing subspace of the pair of doubly commuting isometries $T_{\phi(z)}$ and $T_{\phi(w)}$, by the Wold decomposition of the pair of isometries on $\mathcal{M}$, we have

$$
\widetilde{\mathcal{M}}=\oplus_{l, k \geq 0} \phi(z)^{l} \phi(w)^{k} L_{\widetilde{\mathcal{M}}},
$$

where $L_{\widetilde{\mathcal{M}}}$ is the wandering subspace

$$
L_{\widetilde{\mathcal{M}}}=\operatorname{ker} T_{\phi(z)}^{*} \cap \operatorname{ker} T_{\phi(w)}^{*} \cap \widetilde{\mathcal{M}}
$$

The following theorem gives a complete description of the wandering subspace $L_{\widetilde{\mathcal{M}}}$.

Theorem 2. Suppose that $\mathcal{M}$ is a reducing subspace of $\phi(\mathcal{B})$ orthogonal to $\mathcal{M}_{0}$. If $\left\{e_{1}^{(M)}, \cdots, e_{q_{M}}^{(M)}\right\}$ is a basis of the wandering subspace of $\mathcal{M}$, then

$$
L_{\widetilde{\mathcal{M}}}=\operatorname{span}\left\{e_{1}^{(M)}, \cdots, e_{q_{M}}^{(M)} ; d_{e_{1}^{(M)}}^{1}, \cdots, d_{e_{q_{M}}^{(M)}}^{1}\right\},
$$

and

$$
\operatorname{dim} L_{\widetilde{\mathcal{M}}}=2 q_{M} .
$$

To prove Theorem 2, first we use the Wold decomposition of the pair of doubly commuting isometries $T_{\phi(z)}$ and $T_{\phi(w)}$ on the lifting $\mathcal{K}_{\phi}(=\widetilde{\mathcal{H}})$ of $\mathcal{H}$ to get the dimension of the wandering subspace $\mathcal{L}_{\phi}\left(=L_{\widetilde{\mathcal{H}}}\right)$. By means of the Fredholm theory in [8], we are able to show that the dimension of $\mathcal{L}_{\phi}$ equals $2 N-1$, where $N$ is the order of the Blaschke product $\phi$.

Then by means of the finite dimension of the wandering subspace of these isometries on the reducing subspace we will be able to obtain some structure theorems on reducing subspaces of the multiplication operators by finite Blaschke products on the Bergman space.

Theorem 3. Suppose that $\Omega, \mathcal{M}$ and $\mathcal{N}$ are three distinct nontrivial minimal reducing subspaces contained in $\mathcal{M}_{0}^{\perp}$ for $\phi(\mathcal{B})$. If

$$
\Omega \subset \mathcal{M} \oplus \mathcal{N}
$$

then there is a unitary operator $U: \mathcal{M} \rightarrow \mathcal{N}$ such that $U$ commutes with $\phi(\mathcal{B})$ and $\phi(\mathcal{B})^{*}$. 
The machinery on the Hardy space of the bidisk is not only useful in classifying the reducing subspaces of multiplication operators on the Bergman space, but also it is helpful in understanding the lattice of invariant subspaces of the Bergman shift as in [1] and hence the invariant subspace problem. One of our goals is to develop the Bergman function theory [15], [17] via the Hardy space of the bidisk.

The multiplication operators on the Bergman space is completely different from that in the Hardy space. By the famous Beurling Theorem [9], the lattice of the invariant subspaces of the multiplication operator by $z$ on the Hardy space is completely determined by inner functions. A Beurling's theorem was recently obtained for the Bergman space [1]. On one hand, on the Hardy space, for an inner function $\phi$, the multiplication operator by $\phi$ is a pure isometry and hence unilateral shift (with arbitrary multiplicity). So its reducing subspaces are in one-to-one correspondence with the closed subspaces of $H^{2} \ominus \phi H^{2}$ [5], [16]. Therefore, it has infinitely many reducing subspaces provided that $\phi$ is any inner function other than a Möbius function. Many people have studied the problem of determining reducing subspaces of a multiplication operator on the Hardy space of the unit circle [2], [3] and [20]. For an inner function $\phi$, the multiplication operator by $\phi$ on the Bergman space is a contraction but not an isometry. On the other hand, surprisingly, on the Bergman space, it was shown in [28] and [40] that for a Blaschke product $\phi$ with two zeros, the multiplication operator $M_{\phi}$ has only two nontrivial reducing subspaces. Zhu [40] conjectured that for a Blaschke product $\phi$ with $N$ zeros, the lattice of reducing subspaces of the operator $M_{\phi}$ is generated by $N$ elements. In other words, $M_{\phi}$ has exactly $N$ nontrivial minimal reducing subspaces.

Applying the machinery developed in the paper, we will be able to disproves Zhu's conjecture in the following theorem. For a holomorphic function $\phi$ on the unit disk and a point $c$ in the unit disk, we say that $c$ is a critical point of $\phi$ if its derivative vanishes at $c$.

Theorem 4. Let $\phi$ be a Blaschke product with three zeros. If $\phi(z)$ has a multiple critical point in the unit disk, then $M_{\phi}$ has three nontrivial minimal reducing subspaces. If $\phi$ does not have any multiple critical point in the unit disk, then $M_{\phi}$ has only two nontrivial minimal reducing subspaces.

Bochner's theorem [35], [36] says that every Blaschke product with $N$ zeros has exactly $N-1$ critical points in the unit disk $\mathbb{D}$. Theorem 4 gives a classifcation of reducing subspaces for $M_{\phi}$ for a Blaschke product $\phi$ with three zeros.

Critical points of $\phi$ have important geometric meaning about the self-mapping of the unit disk. The work of Stephonson [24], [25], [26] suggests that the geometric version of the above theorem should be in terms of the Riemann surfaces. A finite Blaschke product $\phi$ with $N$ zeros is an $N$ to 1 conformal map of $\overline{\mathbb{D}}$ onto $\overline{\mathbb{D}}$. Bochner's theorem [35], [36] says that $\phi$ has exactly $N-1$ critical points in the unit disk $\mathbb{D}$ and none on the unit circle. Let $\mathcal{C}$ denote the set of the critical points of $\phi$ in $\mathbb{D}$ and $\mathcal{F}=\phi^{-1} \circ \phi(\mathcal{C})$. Then $\mathcal{F}$ is a finite set, and $\phi^{-1} \circ \phi$ is an $N$-branched analytic function defined and arbitrarily continuable in $\mathbb{D} / \mathcal{F}$. Not all of the branches of $\phi^{-1} \circ \phi$ can be continued to a different branch, for example $z$ is a single valued branch of $\phi^{-1} \circ \phi$. The Riemann surface for $\phi^{-1} \circ \phi$ over $\mathbb{D}$ is an $N$-sheeted cover of $\mathbb{D}$ at most $N(N-1)$ branch points, and it is not connected. The geometric version of Theorem 4 is the following theorem.

Theorem 5. Let $\phi$ be a Blaschke product with three zeros. Then the number of nontrivial minimal reducing subspaces of $M_{\phi}$ equals the number of connected components of the Riemann surface of $\phi^{-1} \circ \phi$ over $\mathbb{D}$.

We would like to point out that there are many essential differences in analysis and geometry between Blaschke products with order three and Blaschke products with order two. On one hand, for Blaschke products $\phi$ with order two, $\phi^{-1} \circ \phi$ contains two analytic functions on the unit disk and hence the Riemann surface for $\phi^{-1} \circ \phi$ over $\mathbb{D}$ is just two copies of the unit disk. On the other hand, for the most Blaschke products with order three, $\phi^{-1} \circ \phi$ has three multivalue functions 
on the unit disk and the Riemann surface for $\phi^{-1} \circ \phi$ over $\mathbb{D}$ has two connected components. This phenomenon makes it difficult for us to classify the reducing subspaces of a multiplication operator with the Blaschke product of order highter than two. It seems that the machinery developed in the paper is inevitable in classifying the reducing subspaces of the multiplication operator by a Blaschke product of higher order.

The problem of determining reducing subspaces of a multiplication operator is equivalent to finding projections in the commutant of the operator which is the set of operators commuting with the multiplication operators. Every von Nuemann algebra is generated by its projections. Theorem 4 says that every von Nuemann algebra contained in the commutant of mulitplication operator by the Blaschke product with third order is commutative. A lot of nice and deep work on the commutant of a multiplication operator has been done on the Hardy space [6], [33], [34] while Blaschke products with finite zeros play an important role. Indeed Cowen proved that for $f \in H^{\infty}$, if the inner factor of $f-f(\alpha)$ is a Blaschke product $\phi$ with finite order for some $\alpha \in \mathbb{D}$, then the commutant of the multiplication operator by $f$ equals the commutant of the multiplication operator by the finite Blaschke product $\phi[6]$. Thus the structure of lattice of reducing subspaces of the multiplication operator by a Blaschke product with finite order is useful in studying the general multiplication operators on the Bergman space.

One of applications of the machinery on the Hardy space of the bidsk is that it was proved in [32] that the multiplication operator on the Bergman space is unitarily equivalent to a weighted unilateral shift operator of finite multiplicity if and only if its symbol is a constant multiple of the $\mathrm{N}$-th power of a Mobius transform. Another one is that we have obtained a complete description of nontrivial minimal reducing subspaces of the multiplication operator by $\phi$ on the Bergman space of the unit disk for the fourth order Blaschke product $\phi$ [31].

Using Theorems 1 and 3, for a finite Blaschke product $\phi$, we are able to show that for two distinct nontrivial minimal reducing subspaces of $\phi(\mathcal{B})$, either they are orthogonal or $\phi(\mathcal{B})$ has two distinct unitarily equivalent reducing subspaces and has also infinitely many minimal reducing subspaces (Theorem 31). Thus either $\phi(\mathcal{B})$ has infinitely many minimal reducing subspaces or the number of nontrivial minimal reducing subspaces of $\phi(\mathcal{B})$ is less than or equal to the order of $\phi$ (Theorem 32). We say that two reducing subspaces $\mathcal{M}$ and $\mathcal{N}$ of $\phi(\mathcal{B})$ are unitarily equivalent if there is a unitary operator $U: \mathcal{M} \rightarrow \mathcal{N}$ such that $U$ commutes with $\phi(\mathcal{B})$ and $\phi(\mathcal{B})^{*}$.

The adjoint of the multiplication operator by a finite Blaschke product is in a Cowen-Douglas class [7]. The theory of Cowen-Douglas classes will be useful in studying the multiplication operators on the Bergman space. On the other hand, we would like to see some applications of the results obtained in the paper to the study of the Cowen-Dougals classes.

We thank R. Douglas for his insightful comments on the relations between multiplication operators and Cowen-Dougals classes, R. Rochberg for his drawing our attention to his papers [10] and [11] with S. Ferguson, K. Stephenson for his drawing our attention to his papers [24], [25], [26] and K. Zhu for his useful comments on his conjecture.

\section{The Wandering subspace of the lifting of the Bergman space}

As pointed out before, we can identify the Bergman space with $\mathcal{H}$. First we introduce notations and show some properties of functions in $\mathcal{H}$. Then we compute the dimension of the wandering space for the lifting $\tilde{\mathcal{H}}$ of $\mathcal{H}$. The dimension is useful for us to find the wandering space for the lifting $\mathcal{M}$ of a reducing subspace $\mathcal{M}$ of $\phi(\mathcal{B})$.

For $\alpha \in \mathbb{D}$, let $k_{\alpha}$ be the reproducing kernel of the Hardy space $H^{2}(\mathbb{T})$ at $\alpha$. That is, for each function $f$ in $H^{2}(\mathbb{T})$,

$$
f(\alpha)=\left\langle f, k_{\alpha}\right\rangle .
$$

In fact, $k_{\alpha}=\frac{1}{(1-\bar{\alpha} z)}$. For $\phi$ in $H^{\infty}(\mathbb{T})$, let $\hat{T}_{\phi}$ denote the analytic Toeplitz operator with symbol $\phi$ on $H^{2}(\mathbb{T})$, given by

$$
\hat{T}_{\phi} h=\phi h .
$$


Thus it is easy to check that

$$
\hat{T}_{\phi}^{*} k_{\alpha}=\overline{\phi(\alpha)} k_{\alpha} .
$$

For an integer $s \geq 0$, let

$$
k_{\alpha}^{s}(z)=\frac{s ! z^{s}}{(1-\bar{\alpha} z)^{s+1}} .
$$

Lemma 6. For each $f \in H^{\infty}(\mathbb{T})$,

$$
\hat{T}_{f}^{*} k_{\alpha}^{s}=\sum_{l=0}^{s} \frac{s !}{l !(s-l) !} \overline{f^{(l)}(\alpha)} k_{\alpha}^{s-l} .
$$

The proof of the above lemma is left for readers. Lemma 6 gives that the kernel of the Toeplitz operator $\hat{T}_{\phi}^{*}$ on the Hardy space of the unit circle is spanned by $\left\{\left\{k_{\alpha_{k}}^{s_{k}}\right\}_{s_{k}=0, \cdots, n_{k}}\right\}_{k=0, \cdots, K}$.

Recall that $\mathcal{H}$ is the subspace of $H^{2}\left(\mathbb{T}^{2}\right)$ spanned by functions $\left\{p_{n}\right\}_{n=0}^{\infty}$. The following two lemmas give some properties for functions in $\mathcal{H}$ or $\mathcal{H}^{\perp}$.

Lemma 7. If $f$ is in $H^{2}\left(\mathbb{T}^{2}\right)$ and continuous on the closed bidisk and $e$ is in $\mathcal{H}$, then

$$
\langle f(z, w), e(z, w)\rangle=\langle f(z, z), e(z, 0)\rangle=\langle f(w, w), e(0, w)\rangle .
$$

The proof of Lemma 7 is left for readers.

Lemma 8. For $h(z, w) \in H^{2}\left(\mathbb{T}^{2}\right), h$ is in $\mathcal{H}^{\perp}$ iff $h(z, z)=0$, for $z \in \mathbb{D}$.

Proof. As pointed out before,

$$
\mathcal{H}^{\perp}=\operatorname{cl}\left\{(z-w) H^{2}\left(\mathbb{T}^{2}\right)\right\} .
$$

Let $z$ be in $\mathbb{D}$. For each function $f(z, w) \in(z-w) H^{2}\left(\mathbb{T}^{2}\right), f(z, z)=0$. Thus $h(z, z)=0$ for each $h \in \mathcal{H}^{\perp}$.

Conversely, assume that for a function $h \in H^{2}\left(\mathbb{T}^{2}\right), h(z, z)=0$, for $z \in \mathbb{D}$. For $0<r<1$, define

$$
h_{r}(z, w)=h(r z, r w) .
$$

Then for each fixed $0<r<1, h_{r}(z, z)=0$, and $h_{r}(z, w)$ is continuous on the closed bidisk and in $H^{2}\left(\mathbb{T}^{2}\right)$.

By Lemma 7 , for each $e(z, w)$ in $\mathcal{H}$,

$$
\left\langle h_{r}(z, w), e(z, w)\right\rangle=\left\langle h_{r}(z, z), e(z, 0)\right\rangle=0 .
$$

On the other hand, by Theorem 3.4.3 in [23],

$$
\langle h(z, w), e(z, w)\rangle=\lim _{r \rightarrow 1^{-}}\left\langle h_{r}(z, w), e(z, w)\right\rangle=0 .
$$

Hence we conclude that $h$ is in $\mathcal{H}^{\perp}$.

The Dirichlet space $\mathcal{D}$ consists of analytic functions on the unit disk whose derivative is in the Bergman space $L_{a}^{2}$.

Theorem 9. For each $f(z, w)$ in $H^{2}\left(T^{2}\right), f$ is in $\mathcal{H}$ if and only if there is a function $\tilde{f}(z)$ in $\mathcal{D}$ such that

for two distinct points $z$ w in the unit disk.

$$
f(z, w)=\frac{\tilde{f}(z)-\tilde{f}(w)}{z-w}
$$

This immediately gives the following three lemmas, which proofs are left for readers.

Lemma 10. Suppose that $e(z, w)$ is in $\mathcal{H}$. If $e(z, z)=0$ for each $z$ in the unit disk, then $e(z, w)=0$ for $(z, w)$ on the torus. 
Lemma 11. If $e(z, w)$ is in $\mathcal{H}$, then

$$
e(z, w)=e(w, z) .
$$

Lemma 12. Suppose $f(z, w)$ is in $\mathcal{H}$. Let $F(z)=f(z, 0)$. Then

$$
f(\lambda, \lambda)=\lambda F^{\prime}(\lambda)+F(\lambda),
$$

for each $\lambda \in \mathbb{D}$.

For an operator $T$ on a Hilbert space, let $\operatorname{ker} T$ denote the kernel of $T$. Then

$$
\operatorname{ker} T^{*}=H \ominus T H .
$$

Given a pure isometry $U$ on a Hilbert space $H$, the classical Wold decomposition theorem [19] states that

$$
H=\oplus_{n \geq 0} U^{n} E,
$$

where $E=H \ominus U H$ is the wandering subspace for $U$ and equals $k e r T^{*}$.

For a function $\phi$ in $H^{\infty}(\mathbb{D})$, we can view $\phi(z)$ and $\phi(w)$ as functions on the torus $\mathbb{T}^{2}$. While $M_{\phi}$ is not an isometry on the Bergman space of the unit disk, the analytic Toeplitz operators $T_{\phi(z)}$ and $T_{\phi(w)}$ are a pair of doubly commuting pure isometries on the Hardy space $H^{2}\left(\mathbb{T}^{2}\right)$ of torus. Since

$$
T_{z}^{*} p_{n}=T_{w}^{*} p_{n}=p_{n-1}
$$

for $n \geq 1$ and

$$
T_{z}^{*} p_{0}=T_{w}^{*} p_{0}=0
$$

$\mathcal{H}$ is an invariant subspace for both $T_{z}^{*}$ and $T_{w}^{*}$. So $\mathcal{H}$ is also an invariant subspace for both $T_{\phi(z)}^{*}$ and $T_{\phi(w)}^{*}$. Recall the lifting $\mathcal{K}_{\phi}$ of $\mathcal{H}$ :

$$
\mathcal{K}_{\phi}=\operatorname{span}\left\{\phi^{l}(z) \phi^{k}(w) \mathcal{H} ; l, k \geq 0\right\} .
$$

Then $\mathcal{K}_{\phi}$ is a reducing subspace for both $T_{\phi(z)}$ and $T_{\phi(w)}$, and so $T_{\phi(z)}$ and $T_{\phi(w)}$ are also a pair of doubly commuting isometries on $\mathcal{K}_{\phi}$.

We consider the Wold decompositions for the pair on both $\mathcal{K}_{\phi}$ and $\mathcal{K}_{\phi}^{\perp}\left(H^{2}\left(\mathbb{T}^{2}\right) \ominus \mathcal{K}_{\phi}\right)$. Introduce wandering subspaces

$$
\mathcal{L}_{\phi}=\operatorname{ker} T_{\phi(z)}^{*} \cap \operatorname{ker} T_{\phi(w)}^{*} \cap \mathcal{K}_{\phi}
$$

and

$$
\widehat{\mathcal{L}_{\phi}}=\operatorname{ker} T_{\phi(z)}^{*} \cap \operatorname{ker} T_{\phi(w)}^{*} \cap \mathcal{K}_{\phi}^{\perp} .
$$

To get the dimension of the wandering subspaces $\mathcal{L}_{\phi}$ and $\widehat{\mathcal{L}_{\phi}}$, we will identify the wandering subspace $\widehat{\mathcal{L}_{\phi}}$ for the Blaschke product $\phi$ with distinct zeros. The following lemma follows from the remark after Lemma 6 about $k e r \hat{T}_{\phi}^{*}$.

Lemma 13. If $\phi(z)$ is a Blaschke product with distinct zeros $\left\{\alpha_{i}\right\}_{i=1}^{N}$, then intersection of the kernel of $T_{\phi(z)}^{*}$ and $T_{\phi(w)}^{*}$ is spanned by $\left\{k_{\alpha_{i}}(z) k_{\alpha_{j}}(w)\right\}_{i, j=1}^{N}$.

The following lemma is implicit in the proof of Theorem 3 [29]. But we give a complete proof of the lemma.

Lemma 14. Suppose that $\phi(z)$ is a Blaschke product with distinct zeros $\left\{\alpha_{i}\right\}_{i=1}^{N}$. Then the wandering space $\widehat{\mathcal{L}_{\phi}}$ is equal to the space spanned by $\left\{k_{\alpha_{i}}(z) k_{\alpha_{j}}(w)-k_{\alpha_{j}}(z) k_{\alpha_{i}}(w): 1 \leq i<j \leq\right.$ $N\}$ and $\left\{\left(T_{z-w}^{*}\left[k_{\alpha_{l}}(z) k_{\alpha_{l+1}}(w)+k_{\alpha_{l+1}}(z) k_{\alpha_{q}}(w)+k_{\alpha_{q}}(z) k_{\alpha_{l}}(w)\right]: 2 \leq l+1<q \leq N\right\}\right.$. Moreover, the dimension of $\widehat{\mathcal{L}_{\phi}}$ equals $(N-1)^{2}$. 
Proof. First we show

Since $\mathcal{H} \subset \mathcal{K}_{\phi}$,

$$
\widehat{\mathcal{L}_{\phi}}=\operatorname{ker} T_{\phi(z)}^{*} \cap \operatorname{ker} T_{\phi(w)}^{*} \cap \mathcal{H}^{\perp}
$$

$$
\widehat{\mathcal{L}_{\phi}} \subset \operatorname{ker} T_{\phi(z)}^{*} \cap \operatorname{ker} T_{\phi(w)}^{*} \cap \mathcal{H}^{\perp} .
$$

Conversely, if $f$ is in $\operatorname{ker} T_{\phi(z)}^{*} \cap \operatorname{ker} T_{\phi(w)}^{*} \cap \mathcal{H}^{\perp}$, then $f$ is in $\operatorname{ker} T_{\phi(z)}^{*} \cap \operatorname{ker} T_{\phi(w)}^{*}$ and orthogonal to $\mathcal{H}$. Thus for each $g(z, w)=\sum_{l, k \geq 0} \phi(z)^{l} \phi(w)^{k} h_{k l} \in \mathcal{K}_{\phi}$ where $h_{k l} \in \mathcal{H}$, we have

$$
\begin{aligned}
\langle f, g\rangle & =\sum_{k, l \geq 0}\left\langle f, \phi(z)^{l} \phi(w)^{k} h_{k l}\right\rangle \\
& =\sum_{k, l \geq 0}\left\langle\left[T_{\phi(z)}^{*}\right]^{l}\left[T_{\phi(w)}^{*}\right]^{k} f, h_{l k}\right\rangle=0 .
\end{aligned}
$$

So $f$ is also in $\widehat{\mathcal{L}_{\phi}}$. Hence we have

$$
\widehat{\mathcal{L}_{\phi}}=\operatorname{ker} T_{\phi(z)}^{*} \cap \operatorname{ker} T_{\phi(w)}^{*} \cap \mathcal{H}^{\perp} .
$$

We are to prove that the dimension of $\widehat{\mathcal{L}_{\phi}}$ is $(N-1)^{2}$. Without loss of generality, we assume that $\alpha_{1}=0$. By Lemma 13, the $N^{2}$ dimensional space $\operatorname{ker} T_{\phi(z)}^{*} \cap \operatorname{ker} T_{\phi(w)}^{*}$ is spanned by $\left\{k_{\alpha_{i}}(z) k_{\alpha_{j}}(w)\right\}_{i, j=1}^{N}$. So it follows from Lemma 8 that $\widehat{\mathcal{L}}_{\phi}$ consists of the elements $h$ in $\operatorname{ker} T_{\phi(z)}^{*} \cap$ $\operatorname{ker} T_{\phi(w)}^{*}$ which satisfy $h(z, z)=0$. That is,

$$
\widehat{\mathcal{L}_{\phi}}=\left\{h=\sum_{i=1}^{N} \sum_{j=1}^{N} c_{i j} k_{\alpha_{i}}(z) k_{\alpha_{j}}(w): h(z, z)=\sum_{i=1}^{N} \sum_{j=1}^{N} c_{i j} k_{\alpha_{i}}(z) k_{\alpha_{j}}(z)=0\right\} .
$$

For any $h \in \widehat{\mathcal{L}}_{\phi}$, taking the limit at infinity and testing the multiplicity at its poles $1 / \bar{\alpha}_{j}$ of the function $h(z, z)$, we immediately have that $h(z, z)=0$ implies $c_{j j}=0, j=1,2 \ldots, N$. That is,

$$
\widehat{\mathcal{L}_{\phi}}=\left\{h=\sum_{i \neq j, i=1}^{N} \sum_{j=1}^{N} c_{i j} k_{\alpha_{i}}(z) k_{\alpha_{j}}(w): h(z, z)=\sum_{i \neq j, i=1}^{N} \sum_{j=1}^{N} c_{i j} k_{\alpha_{i}}(z) k_{\alpha_{j}}(z)=0\right\} .
$$

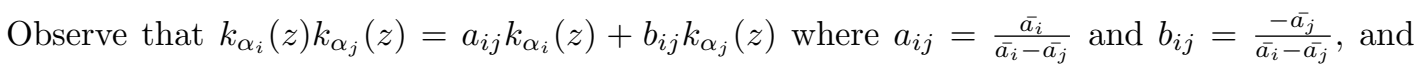
$k_{\alpha_{2}}(z), \ldots, k_{\alpha_{N}}(z)$ are linearly independent. Write $h(z, z)$ as linear combination of $k_{\alpha_{j}}(z), j=$ $2, \ldots, N$, then all the coefficients of $k_{\alpha_{j}}(z)$ must be zero. So we have a system of another $N-1$ linear equations governing $c_{i j}, i \neq j, i, j=1, \ldots, N$. It is easy to check that the rank of the coefficient matrix of the system is $N-1$. Hence the dimension of $\widehat{\mathcal{L}_{\phi}}$ (as the solution space of $N^{2}-N$ unknown variables governed by $N-1$ linearly independent equations) equals $N^{2}-N-$ $(N-1)$. The proof is finished.

We are ready to prove our main result in the section.

Theorem 15. Let $\phi$ be a Blaschke product with $N$ zeros in the unit disk. Then

$$
\mathcal{K}_{\phi}=\oplus_{l, k \geq 0} \phi^{l}(z) \phi^{k}(w) \mathcal{L}_{\phi}
$$

and

$$
H^{2}\left(\mathbb{T}^{2}\right) \ominus \mathcal{K}_{\phi}=\oplus_{l, k \geq 0} \phi^{l}(z) \phi^{k}(w) \widehat{\mathcal{L}}_{\phi} .
$$

The dimension of $\widehat{\mathcal{L}_{\phi}}$ equals $(N-1)^{2}$ and the dimension of $\mathcal{L}_{\phi}$ equals $2 N-1$.

Proof. As pointed out early in this section, $T_{\phi(z)}$ and $T_{\phi(w)}$ are a pair of doubly commuting isometries on both $\mathcal{K}_{\phi}$ and $H^{2}\left(\mathbb{T}^{2}\right) \ominus \mathcal{K}_{\phi}$. Consider the Wold decomposition of $T_{\phi(z)}$ on $\mathcal{K}_{\phi}$ to get

$$
\mathcal{K}_{\phi}=\oplus_{l \geq} \phi(z)^{l} E
$$


where $E$ is the wandering subspace for $T_{\phi(z)}$ given by

$$
\begin{aligned}
E & =\mathcal{K}_{\phi} \ominus\left[T_{\phi(z)} \mathcal{K}_{\phi}\right] \\
& =\operatorname{ker}\left[T_{\phi(z)}^{*} \mid \mathcal{K}_{\phi}\right]=\operatorname{ker} T_{\phi(z)}^{*} \cap \mathcal{K}_{\phi} .
\end{aligned}
$$

Since $T_{\phi(z)}$ and $T_{\phi(w)}$ are doubly commuting, $E$ is a reducing subspace of $T_{\phi(w)}$. Thus $\left.T_{\phi(w)}\right|_{E}$ is still an isometry. The Wold decomposition theorem again gives

$$
E=\oplus_{k \geq 0} \phi(w)^{k} E_{1}
$$

where $E_{1}$ is the wandering subspace for $\left.T_{\phi(w)}\right|_{E}$ given by

$$
\begin{aligned}
E_{1} & =E \ominus T_{\phi(w)} E \\
& =\operatorname{ker} T_{\phi(w)}^{*} \cap E=\operatorname{ker} T_{\phi(z)}^{*} \cap T_{\phi(w)}^{*} \cap \mathcal{K}_{\phi} .
\end{aligned}
$$

This gives

$$
\mathcal{K}_{\phi}=\oplus_{l, k \geq 0} \phi^{l}(z) \phi^{k}(w) \mathcal{L}_{\phi} .
$$

Considering the Wold decompositions of $T_{\phi(z)}$ and $T_{\phi(w)}$ on $H^{2}\left(\mathbb{T}^{2}\right) \ominus \mathcal{K}_{\phi}$, similarly we obtain

$$
H^{2}\left(\mathbb{T}^{2}\right) \ominus \mathcal{K}_{\phi}=\oplus_{l, k \geq 0} \phi^{l}(z) \phi^{k}(w) \widehat{\mathcal{L}_{\phi}} .
$$

Noting

$$
\operatorname{ker} T_{\phi(z)}^{*} \cap \operatorname{ker} T_{\phi(w)}^{*}=\mathcal{L}_{\phi} \oplus \widehat{\mathcal{L}_{\phi}}
$$

we have

$$
\operatorname{dim}\left[\operatorname{ker} T_{\phi(z)}^{*} \cap \operatorname{ker} T_{\phi(w)}^{*}\right]=\operatorname{dim}\left[\mathcal{L}_{\phi}\right]+\operatorname{dim}\left[\widehat{\mathcal{L}_{\phi}}\right]
$$

By Lemma 13, the dimension of $\operatorname{ker} T_{\phi(z)}^{*} \cap \operatorname{ker} T_{\phi(w)}^{*}$ equals $N^{2}$. Hence

$$
\operatorname{dim}\left[\mathcal{L}_{\phi}\right]=N^{2}-\operatorname{dim}\left[\widehat{\mathcal{L}}_{\phi}\right]
$$

To finish the proof, it suffices to show that the dimension of $\widehat{\mathcal{L}_{\phi}}$ equals $(N-1)^{2}$. By Lemma 14, for a Blaschke product $\phi(z)$ with distinct zeros, the dimension of $\widehat{\mathcal{L}_{\phi}}$ equals $(N-1)^{2}$. We need to show that this is still true for a Blaschke product $B$ with $N$ zeros which perhaps contains some repeated zeros. To do so, for a given $\lambda \in \mathbb{D}$, let $\phi_{\lambda}(z)$ be the Möbius transform $\frac{z-\lambda}{1-\bar{\lambda} z}$. Then $\phi_{\lambda} \circ \phi(z)$ is a Blaschke product with $N$ zeros in the unit disk and

$$
T_{\phi_{\lambda} \circ \phi(z)}=\left(T_{\phi(z)}-\lambda I\right)\left(I-\lambda T_{\phi(z)}\right)^{-1} .
$$

Thus $\mathcal{K}_{\phi}=\mathcal{K}_{\phi_{\lambda} \circ \phi}$, and so

$$
\begin{aligned}
\widehat{\mathcal{L}_{\phi_{\lambda} \circ \phi}} & =\operatorname{ker} T_{\phi_{\lambda} \circ \phi(z)}^{*} \cap \operatorname{ker} T_{\phi_{\lambda} \circ \phi(w)}^{*} \cap\left[H^{2}\left(T^{2}\right) \ominus \mathcal{K}_{\phi_{\lambda} \circ \phi}\right] \\
& =\operatorname{ker} T_{\phi(z)-\lambda}^{*} \cap \operatorname{ker} T_{\phi(w)-\lambda}^{*} \cap\left[H^{2}\left(T^{2}\right) \ominus \mathcal{K}_{\phi}\right] .
\end{aligned}
$$

The last equality follows from that

$$
\operatorname{ker} T_{\phi(z)-\lambda}^{*}=\operatorname{ker} T_{\phi_{\lambda} \circ \phi(z)}^{*},
$$

and

We have the fact that

$$
\operatorname{ker} T_{\phi(w)-\lambda}^{*}=\operatorname{ker} T_{\phi_{\lambda} \circ \phi(w)}^{*} .
$$

$$
\operatorname{dim} \widehat{\mathcal{L}_{\phi_{\lambda} \circ \phi}}=-\operatorname{index}\left(T_{\phi(z)-\lambda}^{*}, T_{\phi(w)-\lambda}^{*}\right),
$$

where $\operatorname{index}\left(T_{\phi(z)-\lambda}^{*}, T_{\phi(w)-\lambda}^{*}\right)$ is the Fredholm index of the pair $\left(T_{\phi(z)-\lambda}^{*}, T_{\phi(w)-\lambda}^{*}\right)$, which was first introduced in [8]. The proof of the fact is left for readers. It was shown [8] that the Fredholm index of the pair $\left(T_{\phi(z)-\lambda}^{*}, T_{\phi(w)-\lambda}^{*}\right)$ is a continuous mapping from the set of the Fredholm tuples to the set of integers. Thus for a sufficiently small $\lambda$,

$$
i n d e x\left(T_{\phi(z)-\lambda}^{*}, T_{\phi(w)-\lambda}^{*}\right)=i n d e x\left(T_{\phi(z)}^{*}, T_{\phi(w)}^{*}\right) .
$$


If $\lambda$ is not in the critical values set $\mathcal{C}=\left\{\mu \in \mathbb{D}: \mu=\phi(z)\right.$ and $\phi^{\prime}(z)=0$ for some $\left.z \in \mathbb{D}\right\}$ of $\phi$, then $\phi_{\lambda} \circ \phi(z)$ is a Blaschke product with $N$ distinct zeros in $\mathbb{D}$. In fact, Bochner's theorem implies that there are at most $N-1$ points in $\mathcal{C}$. In this case, by Lemma 14,

$$
\operatorname{dim} \widehat{\mathcal{L}_{\phi_{\lambda} \circ \phi}}=(N-1)^{2} \text {. }
$$

Since the set $\mathcal{C}$ has zero area, we conclude that the dimension of $\widehat{\mathcal{L}}_{\phi}$ equals $(N-1)^{2}$.

\section{BASIC CONSTRUCTIONS}

In this section we will construct a family $\left\{d_{e}^{k}\right\}$ of functions and a function $d_{e}^{0}$ in $\mathcal{L}_{\phi}$ for each $e \in \operatorname{ker} T_{\phi(z)}^{*} \cap \operatorname{ker} T_{\phi(w)}^{*} \cap \mathcal{H}$, which have properties in Theorem 1 in Section 1 to present the proof of Theorem 1 that gives a relation between $d_{e}^{1}$ and $d_{e}^{0}$. The relation is very useful for us to understand the structure of the minimal reducing subspaces in the rest of the paper.

3.1. First Construction. Let $L_{0}$ be $\operatorname{ker} T_{\phi(z)}^{*} \cap \operatorname{ker} T_{\phi(w)}^{*} \cap \mathcal{H}$. It is easy to check that the dimension of $L_{0}$ equals the order of the Blaschke product.

First we will show that for a given reducing subspace $\mathcal{M}$ for $\phi(\mathcal{B})$, for each $e \in \mathcal{M} \cap L_{0}$ and each integer $l \geq 1$, there are a family of functions $\left\{d_{e}^{k}\right\}_{k=1}^{l}$ such that

$$
p_{l}(\phi(z), \phi(w)) e+\sum_{k=0}^{l-1} p_{k}(\phi(z), \phi(w)) d_{e}^{l-k} \in \mathcal{M} .
$$

These functions are useful in studying the structure of the multiplication operator $M_{\phi}$ on the Bergman space.

The following lemma shows that for each reducing subspace $\mathcal{M}$ of $\phi(\mathcal{B})$, the intersection of $\mathcal{M}$ and $L_{0}$ is nontrivial.

Lemma 16. If $\mathcal{M}$ is a nontrivial reducing subspace for $\phi(\mathcal{B})$, then the wandering subspace of $\mathcal{M}$ is contained in $L_{0}$.

Proof. Let $\mathcal{M}$ be a nontrivial reducing subspace for $\phi(\mathcal{B})$. For each $f$ in $\mathcal{H}, \mathcal{P}_{\mathcal{M}} f$ is in $\mathcal{M}$. Thus for each $e$ in the wandering subspace $\mathcal{M} \ominus \phi(\mathcal{B}) \mathcal{M}$ of $\mathcal{M}$,

$$
\begin{aligned}
0 & =\left\langle e, \phi(\mathcal{B}) \mathcal{P}_{\mathcal{M}} f\right\rangle=\left\langle e, \mathcal{P}_{\mathcal{M}} \phi(\mathcal{B}) f\right\rangle \\
& =\langle e, \phi(\mathcal{B}) f\rangle=\left\langle T_{\phi(z)}^{*} e, f\right\rangle .
\end{aligned}
$$

The second equality follows from that $\mathcal{M}$ is a reducing subspace and the last equality follows from the fact that for each $f \in \mathcal{H}$,

$$
\phi(\mathcal{B})^{*} f=T_{\phi(z)}^{*} f=T_{\phi(w)}^{*} f .
$$

So $T_{\phi(z)}^{*} e=0$. Similarily, we also have that $T_{\phi(w)}^{*} e=0$. This gives that $e$ is in $L_{0}$ to complete the proof.

Lemma 17. If $\mathcal{M}$ is a reducing subspace for $\phi(\mathcal{B})$, then $\phi(\mathcal{B})^{*} \mathcal{M}=\mathcal{M}$.

Proof. First note that for a Blaschke product $\phi(z)$ with finite order, $\phi(\mathcal{B})$ is Fredholm and the kernel of $\phi(\mathcal{B})$ contains only zero. Thus

$$
\phi(\mathcal{B})^{*} \mathcal{H}=\mathcal{H}
$$

Suppose that $\mathcal{M}$ is a reducing subspace for $\phi(\mathcal{B})$. Let $\mathcal{N}=\mathcal{M}^{\perp}$. Then

$$
\phi(\mathcal{B})^{*}=\left.\left.\phi(\mathcal{B})^{*}\right|_{\mathcal{M}} \oplus \phi(\mathcal{B})^{*}\right|_{\mathcal{N}}
$$

under the decomposition $\mathcal{H}=\mathcal{M} \oplus \mathcal{N}$. Since $\phi(\mathcal{B})^{*}$ is subjective,

$$
\left.\phi(\mathcal{B})^{*}\right|_{\mathcal{M}} \mathcal{M}=\mathcal{M}
$$

This completes the proof. 
Theorem 18. Suppose that $\mathcal{M}$ is a reducing subspace for $\phi(\mathcal{B})$. For a given $e$ in the wandering subspace of $\mathcal{M}$, there are a unique family of functions $\left\{d_{e}^{k}\right\} \subset \mathcal{L}_{\phi} \ominus L_{0}$ such that

(1) $p_{l}(\phi(z), \phi(w)) e+\sum_{k=0}^{l-1} p_{k}(\phi(z), \phi(w)) d_{e}^{l-k}$ is in $\mathcal{M}$, for each $l \geq 1$.

(2) $P_{\mathcal{H}}\left[p_{l}(\phi(z), \phi(w)) d_{e}^{k}\right]$ is in $\mathcal{M}$ for each $k \geq 1$, and $l \geq 0$.

Proof. For a given $e$ in the wandering subspace of $\mathcal{M}$, first we will use mathematical induction to construct a family of functions $\left\{d_{e}^{k}\right\}$.

By Lemma 16, $e$ is in $L_{0}$. A simple calculation gives $T_{\phi(z)}^{*}[(\phi(z)+\phi(w)) e]=e$, and $T_{\phi(w)}^{*}[(\phi(z)+$ $\phi(w)) e]=e$. By Lemma 17, there is a unique function $\tilde{e} \in \mathcal{M} \ominus L_{0}$ such that

$$
T_{\phi(z)}^{*} \tilde{e}=T_{\phi(w)}^{*} \tilde{e}=e .
$$

This gives

$$
T_{\phi(z)}^{*}[\tilde{e}-(\phi(z)+\phi(w)) e]=e-e=0
$$

and

$$
T_{\phi(w)}^{*}[\tilde{e}-(\phi(z)+\phi(w)) e]=e-e=0,
$$

to get that letting $d_{e}^{1}=\tilde{e}-(\phi(z)+\phi(w)) e, d_{e}^{1}$ is in $\operatorname{ker} T_{\phi(z)}^{*} \cap \operatorname{ker} T_{\phi(w)}^{*}$, and

$$
p_{1}(\phi(z), \phi(w)) e+d_{e}^{1}=(\phi(z)+\phi(w)) e+d_{e}^{1} \in \mathcal{M} .
$$

Because both $\tilde{e}$ and $e$ are in $\mathcal{M}$, we have that $d_{e}^{1}$ is in $\mathcal{K}_{\phi}$, and hence $d_{e}^{1}$ is in $\mathcal{L}_{\phi}$.

Next we show that $d_{e}^{1}$ is orthogonal to $L_{0}$. To do so, let $f \in L_{0}$. A simple calculation gives

$$
\begin{aligned}
\left\langle d_{e}^{1}, f\right\rangle & =\langle\tilde{e}-(\phi(z)+\phi(w)) e, f\rangle \\
& =\langle\tilde{e}, f\rangle-\langle(\phi(z)+\phi(w)) e, f\rangle \\
& =0-\left\langle e, T_{\phi(z)}^{*} f+T_{\phi(w)}^{*} f\right\rangle=0 .
\end{aligned}
$$

The third equality follows from that $\tilde{e}$ is in $\mathcal{M} \ominus L_{0}$. This gives that $d_{e}^{1}$ is in $\mathcal{L}_{\phi} \ominus L_{0}$.

Assume that for $n<l$ there are a family of functions $\left\{d_{e}^{k}\right\}_{k=1}^{n} \subset \mathcal{L}_{\phi} \ominus L_{0}$ such that

$$
p_{n}(\phi(z), \phi(w)) e+\sum_{k=0}^{n-1} p_{k}(\phi(z), \phi(w)) d_{e}^{n-k} \in \mathcal{M} .
$$

Let $E=p_{n}(\phi(z), \phi(w)) e+\sum_{k=0}^{n-1} p_{k}(\phi(z), \phi(w)) d_{e}^{n-k}$. By Lemma 17 again, there is a unique function $\tilde{E}$ in $\mathcal{M} \ominus L_{0}$ such that

$$
T_{\phi(z)}^{*} \tilde{E}=T_{\phi(w)}^{*} \tilde{E}=E .
$$

Let $F=p_{n+1}(\phi(z), \phi(w)) e+\sum_{k=1}^{n} p_{k}(\phi(z), \phi(w)) d_{e}^{n+1-k}$. Since

$$
T_{\phi(z)}^{*}\left[p_{k}(\phi(z), \phi(w)) f\right]=T_{\phi(w)}^{*}\left[p_{k}(\phi(z), \phi(w)) f\right]=p_{k-1}(\phi(z), \phi(w)) f,
$$

for each $f$ in $\mathcal{L}_{\phi}$ and $k \geq 1$, simple calculations give

$$
T_{\phi(z)}^{*} F=T_{\phi(w)}^{*} F=E .
$$

Thus

$$
\begin{aligned}
T_{\phi(z)}^{*}(\tilde{E}-F) & =T_{\phi(w)}^{*}(\tilde{E}-F) \\
& =E-E=0
\end{aligned}
$$


So letting $d_{e}^{n+1}=\tilde{E}-F, d_{e}^{n+1}$ is in $\operatorname{ker} T_{\phi(z)}^{*} \cap \operatorname{ker} T_{\phi(w)}^{*}$. Noting $\tilde{E}$ is orthogonal to $L_{0}$, we have that for each $f \in L_{0}$,

$$
\begin{aligned}
\left\langle d_{e}^{n+1}, f\right\rangle & =\langle\tilde{E}, f\rangle-\langle F, f\rangle \\
& =-\left[\left\langle p_{n+1}(\phi(z), \phi(w)) e, f\right\rangle+\sum_{k=1}^{n}\left\langle p_{k}(\phi(z), \phi(w)) d_{e}^{n+1-k}, f\right\rangle\right] \\
& =0,
\end{aligned}
$$

to get that $d_{e}^{n+1}$ is in $\mathcal{L}_{\phi} \ominus L_{0}$. Hence

$$
p_{n+1}(\phi(z), \phi(w)) e+\sum_{k=1}^{n} p_{k}(\phi(z), \phi(w)) d_{e}^{n+1-k}+d_{e}^{n+1} \in \mathcal{M} .
$$

This gives a family of functions $\left\{d_{e}^{k}\right\} \subset \mathcal{L}_{\phi} \ominus L_{0}$ satifying Property (1).

To finish the proof we need only to show that Property (2) holds. A simple calculation gives

$$
\begin{aligned}
2 \phi(\mathcal{B}) e & =P_{\mathcal{H}}\left(p_{1}(\phi(z), \phi(w)) e\right) \\
& =P_{\mathcal{H}}\left(p_{1}(\phi(z), \phi(w)) e+d_{e}^{1}\right)-P_{\mathcal{H}}\left(d_{e}^{1}\right) \\
& =p_{1}(\phi(z), \phi(w)) e+d_{e}^{1}-P_{\mathcal{H}}\left(d_{e}^{1}\right)
\end{aligned}
$$

This implies

$$
P_{\mathcal{H}}\left(d_{e}^{1}\right)=\left[p_{1}(\phi(z), \phi(w)) e+d_{e}^{1}\right]-2 \phi(\mathcal{B}) e \in \mathcal{M} .
$$

Noting that $\left(d_{e}^{1}-P_{\mathcal{H}} d_{e}^{1}\right)$ is in $\mathcal{H}^{\perp}=[z-w]$ and $[z-w]$ is an invariant subspace for analytic Toeplitz operators, we have that

$$
\left[p_{l-1}(\phi(z), \phi(w))\left(d_{e}^{1}-P_{\mathcal{H}} d_{e}^{1}\right)\right] \in \mathcal{H}^{\perp},
$$

and so

$$
P_{\mathcal{H}}\left[p_{l-1}(\phi(z), \phi(w))\left(d_{e}^{1}-P_{\mathcal{H}} d_{e}^{1}\right)\right]=0
$$

to get

$$
P_{\mathcal{H}}\left[p_{l-1}(\phi(z), \phi(w))\left(d_{e}^{1}\right)\right]=P_{\mathcal{H}}\left\{p_{l-1}(\phi(z), \phi(w))\left[P_{\mathcal{H}} d_{e}^{1}\right]\right\} \in \mathcal{M} .
$$

Assume that $P_{\mathcal{H}}\left[p_{l}(\phi(z), \phi(w)) d_{e}^{k}\right] \in \mathcal{M}$ for $k \leq n$ and any $l \geq 0$. To finish the proof by induction we need only to show that

$$
\left.P_{\mathcal{H}}\left[p_{l}(\phi(z), \phi(w)) d_{e}^{n+1}\right)\right] \in \mathcal{M}
$$

for any $l \geq 0$.

A simple calculation gives

$$
\begin{aligned}
(n+2) \phi(\mathcal{B})^{n+1} e & =P_{\mathcal{H}}\left[p_{n+1}(\phi(z), \phi(w)) e+\sum_{k=0}^{n} p_{k}(\phi(z), \phi(w)) d_{e}^{n+1-k}\right] \\
& -\left\{P_{\mathcal{H}}\left[d_{e}^{n+1}\right]+P_{\mathcal{H}}\left[\sum_{k=1}^{n} p_{k}(\phi(z), \phi(w)) d_{e}^{n+1-k}\right]\right\} .
\end{aligned}
$$

Thus

$$
\begin{aligned}
P_{\mathcal{H}}\left[d_{e}^{n+1}\right] & =P_{\mathcal{H}}\left[p_{n+1}(\phi(z), \phi(w)) e+\sum_{k=0}^{n} p_{k}(\phi(z), \phi(w)) d_{e}^{n+1-k}\right]- \\
& \left\{(n+2) \phi(\mathcal{B})^{n+1} e+P_{\mathcal{H}}\left[\sum_{k=1}^{n} p_{k}(\phi(z), \phi(w)) d_{e}^{n+1-k}\right]\right\} .
\end{aligned}
$$


Property (1) gives that the first term in the last equality is $\mathcal{M}$, the induction hypothesis gives that the last term is in $\mathcal{M}$ and the second term belongs to $\mathcal{M}$ since $e \in \mathcal{M}$ and $\mathcal{M}$ is a reducing subspace for $\phi(\mathcal{B})$. So $P_{\mathcal{H}}\left[d_{e}^{n+1}\right]$ is in $\mathcal{M}$. Therefore we conclude

$$
P_{\mathcal{H}}\left[p_{l}(\phi(z), \phi(w)) d_{e}^{n+1}\right]=P_{\mathcal{H}}\left[\left(p_{l}(\phi(z), \phi(w))\left(P_{\mathcal{H}} d_{e}^{n+1}\right)\right] \in \mathcal{M},\right.
$$

to complete the proof.

In the special case for $\mathcal{H}$, as $\mathcal{H}$ is a reducing subspace for $\phi(\mathcal{B})$, Theorem 18 immediately gives the following theorem.

Theorem 19. For a given $e \in L_{0}$ there are a unique family of functions $\left\{d_{e}^{k}\right\} \subset \mathcal{L}_{\phi} \ominus L_{0}$ such that

for each $l \geq 1$.

$$
p_{l}(\phi(z), \phi(w)) e+\sum_{k=0}^{l-1} p_{k}(\phi(z), \phi(w)) d_{e}^{l-k} \in \mathcal{H}
$$

3.2. Second Construction. Next for a given $e \in L_{0}$, we will show that the function $d_{e}^{0}(z, w)$ given by

is in $\mathcal{L}_{\phi}$ and satisfies

$$
d_{e}^{0}(z, w)=w e(0, w) e_{0}(z, w)-w \phi_{0}(w) e(z, w)
$$

for each $l \geq 1$.

$$
p_{l}(\phi(z), \phi(w)) e+p_{l-1}(\phi(z), \phi(w)) d_{e}^{0} \in \mathcal{H}
$$

Recall that $\phi$ is a Blaschke product with zeros $\left\{\alpha_{k}\right\}_{0}^{K}$ and $\alpha_{k}$ repeats $n_{k}+1$ times, and $\phi(z)=z \phi_{0}(z)$ where $\phi_{0}$ is a Blaschke product with $N-1$ zeros. Let $e_{0}=\frac{\phi(z)-\phi(w)}{z-w}$. Theorem 9 gives that $e_{0}$ is in $\mathcal{H}$ since $\phi$ is a Blaschke product with finite order. This also gives that $e_{0}(z, 0)=\phi_{0}(z)$.

Theorem 20. Let $f$ be a nonzero function $f$ in $\mathcal{H} . p_{l}(\phi(z), \phi(w)) f \in \mathcal{H}$, for some $l \geq 1$ if and only if $f=\lambda e_{0}$ for some constant $\lambda$

The proof of Theorem 20 is left for readers.

Theorem 20 gives that

$$
\mathcal{M}_{0}=\operatorname{span}_{l \geq 0}\left\{p_{l}(\phi(z), \phi(w)) e_{0}\right\}
$$

is a reducing subspace of $\phi(\boldsymbol{B})$. We will study the space in next section.

For each $e(z, w)$ in $L_{0}$, let

$$
d_{e}^{0}(z, w)=w e(0, w) e_{0}(z, w)-w \phi_{0}(w) e(z, w) .
$$

Theorem 21. For each $e(z, w)$ in $L_{0}, d_{e}^{0}(z, w)$ is a function in $\mathcal{L}_{\phi}$ such that

$$
p_{l}(\phi(z), \phi(w)) e+p_{l-1}(\phi(z), \phi(w)) d_{e}^{0} \in \mathcal{H}
$$

for $l \geq 1$.

Proof. First we show that the function $d_{e}^{0}(z, w)$ is in $\operatorname{ker} T_{\phi(z)}^{*} \cap \operatorname{ker} T_{\phi(w)}^{*}$. To do this, by Theorem 9 , write

$$
e(z, w)=\frac{\phi_{e}(z)-\phi_{e}(w)}{z-w}
$$

for some function $\phi_{e}$ in the Dirichlet space $\mathcal{D}$ with $\phi_{e}(0)=0$. Letting $w=0$ in the above equality gives that $e(z, 0)=e(0, z)=\bar{z} \phi_{e}(z)$.

$$
\begin{aligned}
d_{e}^{0}(z, w) & =w e(0, w) e_{0}(z, w)-w \phi_{0}(w) e(z, w) \\
& =\phi_{e}(w)\left[\frac{\phi(z)-\phi(w)}{z-w}\right]-\phi(w)\left[\frac{\phi_{e}(z)-\phi_{e}(w)}{z-w}\right] \\
& =\frac{\phi_{e}(w) \phi(z)-\phi(w) \phi_{e}(z)}{z-w} .
\end{aligned}
$$


This gives that $d_{e}^{0}(z, w)$ is a symmetric function of $z$ and $w$. Since $e_{0}(z, w)$ and $e(z, w)$ are symmetric functions of $z$ and $w$ in $L_{0}$, we have

$$
\begin{aligned}
T_{\phi(z)}^{*}\left[d_{e}^{0}(z, w)\right] & =T_{\phi(z)}^{*}\left[w e(0, w) e_{0}(z, w)-w \phi_{0}(w) e(z, w)\right] \\
& =w e(0, w) T_{\phi(z)}^{*}\left[e_{0}(z, w)\right]-w \phi_{0}(w) T_{\phi(z)}^{*} e(z, w)=0 \\
T_{\phi(w)}^{*}\left[d_{e}^{0}(z, w)\right] & =T_{\phi(w)}^{*}\left[d_{e}^{0}(w, z)\right] \\
& =T_{\phi(w)}^{*}\left[z e(0, z) e_{0}(w, z)-z \phi_{0}(z) e(w, z)\right] \\
& =z e(0, z) T_{\phi(w)}^{*}\left[e_{0}(z, w)\right]-z \phi_{0}(z) T_{\phi(w)}^{*} e(z, w)=0
\end{aligned}
$$

to get that $d_{e}^{0}(z, w)$ is in $\operatorname{ker} T_{\phi(z)}^{*} \cap \operatorname{ker} T_{\phi(w)}^{*}$.

Next we show that $d_{e}^{0}(z, w)$ satisfies $(3)$. To do this, let

$$
E_{l}=p_{l}(\phi(z), \phi(w)) e+p_{l-1}(\phi(z), \phi(w)) d_{e}^{0} .
$$

We show that

$$
E_{l}=\frac{\phi_{e}(z) \phi^{l}(z)-\phi_{e}(w) \phi^{l}(w)}{z-w} .
$$

By Theorem 9, this gives that $E_{l}$ is in $\mathcal{H}$. Simple calculations give

$$
\begin{aligned}
p_{l}(\phi(z), \phi(w)) e & =\left[\frac{\phi^{l+1}(z)-\phi^{l+1}(w)}{\phi(z)-\phi(w)}\right]\left[\frac{\phi_{e}(z)-\phi_{e}(w)}{z-w}\right] \\
& =\frac{\phi^{l+1}(z) \phi_{e}(z)-\phi^{l+1}(z) \phi_{e}(w)-\phi^{l+1}(w) \phi_{e}(z)+\phi^{l+1}(z) \phi_{e}(w)}{(\phi(z)-\phi(w))(z-w)}
\end{aligned}
$$

and

$$
\begin{aligned}
p_{l-1}(\phi(z), \phi(w)) d_{e}^{0} & =\left[\frac{\phi^{l}(z)-\phi^{l}(w)}{\phi(z)-\phi(w)}\right]\left[\frac{\phi_{e}(w) \phi(z)-\phi(w) \phi_{e}(z)}{z-w}\right] \\
& =\frac{\phi^{l+1}(z) \phi_{e}(w)-\phi^{l}(z) \phi(w) \phi_{e}(z)-\phi^{l}(w) \phi(z) \phi_{e}(w)+\phi^{l+1}(w) \phi_{e}(z)}{(\phi(z)-\phi(w))(z-w)} .
\end{aligned}
$$

Thus

$$
\begin{aligned}
E_{l} & =p_{l}(\phi(z), \phi(w)) e+p_{l-1}(\phi(z), \phi(w)) d_{e}^{0} \\
& =\frac{\phi^{l}(z) \phi_{e}(z)(\phi(z)-\phi(w))-\phi^{l}(w) \phi_{e}(w)(\phi(z)-\phi(w))}{(\phi(z)-\phi(w))(z-w)} \\
& =\frac{\phi_{e}(z) \phi^{l}(z)-\phi_{e}(w) \phi^{l}(w)}{z-w} .
\end{aligned}
$$

Since $p_{1}(\phi(z), \phi(w)) e+d_{e}^{0}$ is in $\mathcal{H}$ and $p_{1}(\phi(z), \phi(w)) e$ is in $\mathcal{K}_{\phi}$, we conclude that $d_{e}^{0}$ is in $\mathcal{K}_{\phi}$. Hence $d_{e}^{0}$ is in $\mathcal{L}_{\phi}$. This completes the proof.

Now we are ready to prove Theorem 1.

Proof of Theorem 1. Since $\mathcal{M}$ is orthogonal to $\mathcal{M}_{0}$, we have

$$
\begin{aligned}
\mathcal{H} & =\mathcal{M}_{0} \oplus \mathcal{M}_{0}^{\perp} \\
& =\mathcal{M}_{0} \oplus \mathcal{M} \oplus\left[\mathcal{M}_{0}^{\perp} \cap \mathcal{M}^{\perp}\right]
\end{aligned}
$$

Thus

$$
L_{0}=C e_{0} \oplus\left[\mathcal{M} \cap L_{0}\right] \oplus\left[\mathcal{M}_{0}^{\perp} \cap \mathcal{M}^{\perp} \cap L_{0}\right] .
$$

So $e$ is orthogonal to $e_{0}$, and

$$
L_{0} \ominus e_{0}=\left[\mathcal{M} \cap\left(L_{0} \ominus e_{0}\right)\right] \oplus\left[\mathcal{M}_{0}^{\perp} \cap \mathcal{M}^{\perp} \cap\left(L_{0} \ominus e_{0}\right)\right] .
$$

Let $P_{0}$ denote the orthogonal projection from $H^{2}\left(\mathbb{T}^{2}\right)$ onto the space $C e_{0}$. Let $d_{e}=d_{e}^{0}-P_{0} d_{e}^{0}$. Then $d_{e}$ is orthogonal to $e_{0}$. Theorems 20 and 21 give

$$
p_{l}(\phi(z), \phi(w)) e+p_{l-1}(\phi(z), \phi(w)) d_{e} \in \mathcal{H}
$$


for $l \geq 1$. By Theorem 19, there is a function $d_{e}^{k} \in \mathcal{L}_{\phi} \ominus L_{0}$ such that

$$
p_{l}(\phi(z), \phi(w)) e+\sum_{k=0}^{l-1} p_{k}(\phi(z), \phi(w)) d_{e}^{l-k} \in \mathcal{M},
$$

for each $l \geq 1$. Thus

$$
d_{e}-d_{e}^{1}=p_{1}(\phi(z), \phi(w)) e+d_{e}-\left(p_{1}(\phi(z), \phi(w)) e+d_{e}^{1}\right) \in \mathcal{H} .
$$

So $d_{e}-d_{e}^{1}$ is in $L_{0} \ominus e_{0}$. Write

$$
d_{e}-d_{e}^{1}=e^{\prime}+e^{\prime \prime}
$$

for $e^{\prime} \in \mathcal{M} \cap\left(L_{0} \ominus e_{0}\right)$ and $e^{\prime \prime} \in \mathcal{M}^{\perp} \cap\left(L_{0} \ominus e_{0}\right)$. Thus (5) gives that the following function is in $\mathcal{H}$ :

$$
\begin{aligned}
& p_{2}(\phi(z), \phi(w)) e+p_{1}(\phi(z), \phi(w)) d_{e} \\
=\quad & {\left[p_{2}(\phi(z), \phi(w)) e+p_{1}(\phi(z), \phi(w)) d_{e}^{1}+d_{e}^{2}\right]+\left[p_{1}(\phi(z), \phi(w)) e^{\prime}+d_{e^{\prime}}^{1}\right] } \\
& +\left[p_{1}(\phi(z), \phi(w)) e^{\prime \prime}+d_{e^{\prime \prime}}^{1}\right]-\left(d_{e}^{2}+d_{e^{\prime}}^{1}+d_{e^{\prime \prime}}^{1}\right) .
\end{aligned}
$$

Theorem 18 gives that the first term and the second term in the right hand side are in $\mathcal{M}$ and the third term is in $\mathcal{M}^{\perp}$. Thus the last term must be in $\mathcal{H}$ and hence

$$
d_{e}^{2}+d_{e^{\prime}}^{1}+d_{e^{\prime \prime}}^{1} \in \mathcal{H} \cap \operatorname{ker} T_{\phi(z)}^{*} \cap T_{\phi(w)}^{*}=L_{0} .
$$

By Theorem 18 again, we have

$$
d_{e}^{2}+d_{e^{\prime}}^{1}+d_{e^{\prime \prime}}^{1} \in \mathcal{L}_{\phi} \ominus L_{0}
$$

to get

$$
d_{e}^{2}+d_{e^{\prime}}^{1}+d_{e^{\prime \prime}}^{1}=0
$$

This gives

$$
P_{\mathcal{H}} d_{e^{\prime \prime}}^{1}=-\left(P_{\mathcal{H}} d_{e^{\prime}}^{1}+P_{\mathcal{H}} d_{e}^{2}\right)
$$

On the other hand, Theorem 18 gives $P_{\mathcal{H}} d_{e^{\prime}}^{1}+P_{\mathcal{H}} d_{e}^{2}$ is in $\mathcal{M}$ and $P_{\mathcal{H}^{\prime \prime}} d_{e^{\prime \prime}}^{1}$ is in $\mathcal{M}^{\perp}$. Thus $P_{\mathcal{H}} d_{e^{\prime \prime}}^{1}=0$, and so simple calculations give

$$
\begin{aligned}
\left\|d_{e^{\prime \prime}}^{1}\right\|^{2} & =\left\langle d_{e^{\prime \prime}}^{1}, d_{e^{\prime \prime}}^{1}\right\rangle \\
& =\left\langle d_{e^{\prime \prime}}^{1}, p_{1}(\phi(z), \phi(w)) e^{\prime \prime}+d_{e^{\prime \prime}}^{1}\right\rangle \\
& =\left\langle d_{e^{\prime \prime}}^{1}, P_{\mathcal{H}}\left[p_{1}(\phi(z), \phi(w)) e^{\prime \prime}+d_{e^{\prime \prime}}^{1}\right]\right\rangle \\
& =\left\langle P_{\mathcal{H}}\left(d_{e^{\prime \prime}}^{1}\right), p_{1}(\phi(z), \phi(w)) e^{\prime \prime}+d_{e^{\prime \prime}}^{1}\right\rangle=0 .
\end{aligned}
$$

Hence we have that $d_{e^{\prime \prime}}^{1}=0$, to get

$$
p_{1}(\phi(z), \phi(w)) e^{\prime \prime} \in \mathcal{H} .
$$

Theorem 20 gives that $e^{\prime \prime}=\lambda e_{0}$, for some constant $\lambda$. Since $e^{\prime \prime} \in \mathcal{M}^{\perp} \cap\left(L_{0} \ominus e_{0}\right)$ we conclude that $e^{\prime \prime}=0$ to get $d_{e}=d_{e}^{1}+e^{\prime}$. Letting $\tilde{e}=-e^{\prime} \in \mathcal{M}$, we obtain

$$
\begin{aligned}
d_{e}^{1} & =d_{e}+\tilde{e} \\
& =d_{e}^{0}-P_{0} d_{e}^{0}+\tilde{e} \\
& =d_{e}^{0}+\tilde{e}+\lambda e_{0},
\end{aligned}
$$

as desired. The last equality follows from that $P_{0} d_{e}^{0}=-\lambda e_{0}$ for some constant. This completes the proof. 


\section{The Distinguished REDUCING SUBSPACE}

Theorems 1 and 19 are useful in studying reducing subspaces of $\phi(\mathcal{B})$. In this section we will use them to show that there always exists a unique reducing subspace $\mathcal{M}_{0}$ for $\phi(\mathcal{B})$ such that the restriction of $\phi(\mathcal{B})$ on $\mathcal{M}_{0}$ is unitarily equivalent to the Bergman shift. The existence of such reducing subspace is the main result in [18]. Moreover, we will show that such reducing space is unique. We call $\mathcal{M}_{0}$ to be the distinguished reducing subspace for $\phi(\mathcal{B})$. In fact, $\mathcal{M}_{0}$ is unitarily equivalent the subspace $\operatorname{span}\left\{\phi^{\prime} \phi^{m}: m=0, \cdots, n, \cdots\right\}$ of the Bergman space [27] if $\phi(0)=0$. Furthermore we will show that only the multiplication operator by a finite Blashcke product has such nice property.

Assume that $\phi$ be a Blaschke product of order $N$ with $\phi(0)=0$. Recall $e_{0}(z, w)=\frac{\phi(z)-\phi(w)}{z-w}$. The following lemmas will be used in the proofs of Theorems 25 and 26 . The proofs of those lemmas are left for readers.

Lemma 22. Let $f$ be a function in $H^{2}\left(\mathbb{T}^{2}\right)$. Then

$$
P_{\mathcal{H}}\left[\phi(z) p_{n}(\phi(z), \phi(w)) f\right]=\frac{n+1}{n+2} P_{\mathcal{H}}\left[p_{n+1}(\phi(z), \phi(w)) f\right] .
$$

Lemma 23. Let $\phi(z)$ be an inner function satisfying $\frac{\phi(z)-\phi(w)}{z-w} \in H^{2}\left(\mathbb{T}^{2}\right)$, then

$$
\frac{\phi(z)-\phi(w)}{z-w} \perp \phi(z) H^{2}\left(\mathbb{T}^{2}\right) .
$$

Lemma 24. For an inner function $\phi(z), \frac{\phi(z)-\phi(w)}{z-w}$ is in $H^{2}\left(\mathbb{T}^{2}\right)$ iff $\phi(z)$ is a finite Blaschke product. Moreover, for a Blaschke product $\phi$ of order $N$,

$$
\left\|e_{0}\right\|^{2}=N \text {. }
$$

Now we are ready to prove the first main result in this section.

Theorem 25. Let $\phi$ be a Blaschke product of order $N$. There is a unique reducing subspace $\boldsymbol{\mathcal { M }}_{0}$ for $\phi(\mathcal{B})$ such that $\left.\phi(\mathcal{B})\right|_{M_{0}}$ is unitarily equivalent to the Bergman shift. In fact,

$$
\mathcal{M}_{0}=\operatorname{span}_{l \geq 0}\left\{p_{l}(\phi(z), \phi(w)) e_{0}\right\},
$$

and $\left\{\frac{p_{l}(\phi(z), \phi(w)) e_{0}}{\sqrt{l+1} \sqrt{N}}\right\}_{0}^{\infty}$ form an orthonormal basis of $\mathcal{M}_{0}$.

Proof. First we show that there exists a reducing subspace $\mathcal{M}_{0}$ of $\phi(\mathcal{B})$ such that $\left.\phi(\mathcal{B})\right|_{\mathrm{M}_{0}}$ is unitarily equivalent to the Bergman shift.

Let

$$
\mathcal{M}_{0}=\operatorname{span}_{l \geq 0}\left\{p_{l}(\phi(z), \phi(w)) e_{0}\right\} .
$$

As pointed out before, Theorem 20 gives that $\mathcal{M}_{0}$ is a reducing subspace of $\phi(\mathcal{B})$. Here $e_{0}(z, w)=$ $\frac{\phi(z)-\phi(w)}{z-w}$.

A simple calculation gives

$$
\left\|p_{l}(\phi(z), \phi(w)) e_{0}\right\|_{2}^{2}=(l+1)\left\|e_{0}\right\|_{2}^{2},
$$

and

$$
\left\langle p_{l}(\phi(z), \phi(w)) e_{0}, p_{n}(\phi(z), \phi(w)) e_{0}\right\rangle=0,
$$

for $n \neq l$. Let $E_{n}=\frac{p_{n}(\phi(z), \phi(w)) e_{0}}{\sqrt{(n+1)}\left\|e_{0}\right\|_{2}}$. Thus $\left\{E_{n}\right\}$ are an orthonormal basis of $\mathcal{M}_{0}$. By Lemma 22 we have

$$
\begin{aligned}
\phi(\mathcal{B})\left[p_{n}(\phi(z), \phi(w)) e_{0}\right] & =P_{\mathcal{H}}\left[\phi(z) p_{n}(\phi(z), \phi(w)) e_{0}\right] \\
& =P_{\mathcal{H}}\left[\frac{n+1}{n+2} p_{n+1}(\phi(z), \phi(w)) e_{0}\right] \\
& =\frac{n+1}{n+2} p_{n+1}(\phi(z), \phi(w)) e_{0}
\end{aligned}
$$


to obtain

$$
\begin{aligned}
\phi(\mathcal{B}) E_{n} & =\frac{\phi(\mathcal{B})\left[p_{n}(\phi(z), \phi(w)) e_{0}\right]}{\sqrt{(n+1)}\left\|e_{0}\right\|_{2}} \\
& =\frac{n+1}{n+2} \frac{p_{n+1}(\phi(z), \phi(w)) e_{0}}{\sqrt{(n+1)}\left\|e_{0}\right\|_{2}}=\sqrt{\frac{n+1}{n+2}} E_{n+1} .
\end{aligned}
$$

Clearly, $\phi(\mathcal{B})^{*} E_{0}=0$. This implies that $\left.\phi(\mathcal{B})\right|_{\mathcal{M}_{0}}$ is unitarily equivalent to the Bergman shift.

Suppose that $\mathcal{M}_{1}$ is a reducing subspace of $\phi(\mathcal{B})$ and $\left.\phi(M)\right|_{\mathcal{M}_{1}}$ is unitarily equivalent to the Bergman shift, i.e., there is an orthonormal basis $\left\{F_{n}\right\}$ of $\mathcal{M}_{1}$ such that

$$
\phi(\mathcal{B}) F_{n}=\sqrt{\frac{n+1}{n+2}} F_{n+1} .
$$

Next we will show that $\mathcal{M}_{1}=\mathcal{M}_{0}$. Observe

$$
P_{\mathcal{H}}\left[(\phi(z)+\phi(w)) F_{0}\right]=2 \phi(\mathcal{B}) F_{0}=\frac{2}{\sqrt{2}} F_{1} .
$$

Thus

$$
\left\|P_{\mathcal{H}}\left[(\phi(z)+\phi(w)) F_{0}\right]\right\|^{2}=2
$$

Since

$$
T_{\phi(z)}^{*} F_{0}=\phi(\mathcal{B})^{*} F_{0}=0
$$

a simple calculation gives

$$
\begin{aligned}
\left\|(\phi(z)+\phi(w)) F_{0}\right\|^{2}= & \left\langle(\phi(z)+\phi(w)) F_{0},(\phi(z)+\phi(w)) F_{0}\right\rangle \\
= & \left\langle\phi(z) F_{0}, \phi(z) F_{0}\right\rangle+\left\langle\phi(w) F_{0}, \phi(w) F_{0}\right\rangle \\
& \quad+\left\langle\phi(z) F_{0}, \phi(w) F_{0}\right\rangle+\left\langle\phi(w) F_{0}, \phi(z) F_{0}\right\rangle \\
= & 2\left\langle F_{0}, F_{0}\right\rangle=2 .
\end{aligned}
$$

Thus we obtain

because

$$
P_{\mathcal{H}^{\perp}}\left[(\phi(z)+\phi(w)) F_{0}\right]=0
$$

$$
\left\|(\phi(z)+\phi(w)) F_{0}\right\|^{2}=\left\|P_{\mathcal{H}}\left[(\phi(z)+\phi(w)) F_{0}\right]\right\|^{2}+\left\|P_{\mathcal{H}^{\perp}}\left[(\phi(z)+\phi(w)) F_{0}\right]\right\|^{2} .
$$

So $p_{1}(\phi(z), \phi(w)) F_{0}=(\phi(z)+\phi(w)) F_{0}$ is in $\mathcal{H}$. Theorem 20 gives that $F_{0}=\lambda e_{0}$ for some constant $\lambda$. Thus $\mathcal{M}_{0}$ is a subspace of $\mathcal{M}_{1}$ but $\mathcal{M}_{0}$ is a reducing subspace of $\left.\phi(\mathcal{B})\right|_{\mathcal{M}_{1}}$, which is unitarily equivalent to the Bergman shift. But the Bergman shift is irreducible. So we conclude that $\mathcal{M}_{1}=\mathcal{M}_{0}$, to complete the proof.

For $\phi(z) \in H^{\infty}(\mathbb{D})$, let $S_{\phi}$ denote $P_{\mathcal{H}} M_{\phi} \mid \mathcal{H}$. Then

$$
U^{*} S_{\phi} U=M_{\phi}
$$

where $M_{\phi}$ is the multiplication operator on $L_{a}^{2}(\mathbb{D})$. Indeed, for each $g \in \mathcal{H}$ and any $z \in \mathbb{D}$, we have

$$
\begin{aligned}
\left(U^{*} S_{\phi} g\right)(z) & =\left(S_{\phi} g\right)(z, z) \\
& =\left(P_{\mathcal{H}} \phi g\right)(z, z) \\
& =\left(\phi g-P_{\left.\mathcal{H}^{\perp} \phi g\right)(z, z)}\right. \\
& =\phi(z) g(z, z)=\left(M_{\phi} U^{*} g\right)(z) .
\end{aligned}
$$

The last equality follows from Lemma 8. This gives that $U^{*} S_{\phi}=M_{\phi} U^{*}$. Thus $U^{*} S_{\phi} U=M_{\phi}$.

Theorem 25 tells us that for each finite Blaschke product $\phi, M_{\phi}$ has a unique the distinguished reducing subspace. The following theorem shows that only a multiplication operator by a finite Blaschke product has such property. 
Theorem 26. Let $\phi \in H^{\infty}(\mathbb{D})$. Then $M_{\phi}$ acting on $L_{a}^{2}(\mathbb{D})$ has the distinguished reducing subspace iff $\phi$ is a finite Blaschke product.

Proof. We only need to prove that if $M_{\phi}$ has the distinguished reducing subspace, then $\phi$ is a finite Blaschke product. Now, assume $M_{\phi}$ has the distinguished reducing subspace $\mathcal{M}$ such that $M_{\phi} \mid \mathcal{M}$ is unitarily equivalent to the Bergman shift $M_{z}$, that is, there exists a unitary operator $U: M \rightarrow L_{a}^{2}(\mathbb{D})$ such that $U^{*} M_{z} U=\left.M_{\phi}\right|_{\mathcal{M}}$. Let $K_{\lambda}^{\mathcal{M}}$ be the reproducing kernel of $\mathcal{M}$ for $\lambda \in \mathbb{D}$. Clearly, $K_{\lambda}^{\mathcal{M}} \neq 0$, except for at most a countable set. Thus we have

$$
\begin{aligned}
\mid\left\langle\mathcal{M}_{\phi} K_{\lambda}^{\mathcal{M}}, K_{\lambda}^{\mathcal{M}}\right\rangle & =|\phi(\lambda)|\left\|K_{\lambda}^{\mathcal{M}}\right\|^{2} \\
& =\left|\left\langle M_{z} U K_{\lambda}^{\mathcal{M}}, U K_{\lambda}^{\mathcal{M}}\right\rangle\right| \\
& \leq\left\|M_{z}\right\|\left\|U K_{\lambda}^{\mathcal{M}}\right\|^{2} \leq\left\|K_{\lambda}^{\mathcal{M}}\right\|^{2}
\end{aligned}
$$

to get that $|\phi(\lambda)| \leq 1$ except for at most a countable set. So $\|\phi\|_{\infty} \leq 1$. Since $S_{\phi}$ acting on $\mathcal{H}=H^{2}\left(\mathbb{T}^{2}\right) \ominus[z-w]$ is unitarily equivalent to $M_{\phi}$ acting on $L_{a}^{2}(\mathbb{D})$, this means that $S_{\phi}$, restricted on its corresponding reducing subspace $\mathcal{N}$, is unitarily equivalent to $M_{z}$ acting on $L_{a}^{2}(\mathbb{D})$, that is, there exists a unitary operator $V: \mathcal{N} \rightarrow L_{a}^{2}(\mathbb{D})$ such that $V^{*} M_{z} V=\left.S_{\phi}\right|_{\mathcal{N}}$. Set $e_{n}=V^{*} e_{n}^{\prime}$, where $e_{n}^{\prime}=\sqrt{n+1} z^{n}$ for $n=0,1, \cdots$. Then $S_{\phi}^{*} e_{0}=0$, and hence $M_{\phi(z)}^{*} e_{0}=0$ and $M_{\phi(w)}^{*} e_{0}=0$, where $M_{\phi(z)}$ and $M_{\phi(w)}$ are the operators acting on $H^{2}\left(\mathbb{T}^{2}\right)$. Noticing $S_{\phi(z)}=S_{\phi(w)}$, we have

$$
\left\|V S_{(\phi(z)+\phi(w))} e_{0}\right\|^{2}=\|z+z\|^{2}=2
$$

to obtain

$$
\left\langle\phi(z) e_{0}, \phi(w) e_{0}\right\rangle=\left\langle M_{\phi(w)}^{*} e_{0}, M_{\phi(z)}^{*} e_{0}\right\rangle=0
$$

Thus

$$
\left\|(\phi(z)+\phi(w)) e_{0}\right\|^{2}=\left\|\phi(z) e_{0}\right\|^{2}+\left\|\phi(w) e_{0}\right\|^{2} \leq 2 .
$$

Since

$$
2=\left\|V S_{(\phi(z)+\phi(w))} e_{0}\right\|^{2}=\left\|V P_{\mathcal{H}}(\phi(z)+\phi(w)) e_{0}\right\|^{2}=\left\|P_{\mathcal{H}}(\phi(z)+\phi(w)) e_{0}\right\|^{2},
$$

we have

to obtain

$$
(\phi(z)+\phi(w)) e_{0} \in \mathcal{H}
$$

$$
e_{0}=c \frac{\phi(z)-\phi(w)}{z-w}
$$

for some constant $c$. This follows from Theorem 20 .

On the other hand,

$$
\left\|(\phi(z)+\phi(w)) e_{0}\right\|^{2}=\left\|\phi(z) e_{0}\right\|^{2}+\left\|\phi(w) e_{0}\right\|^{2}=2 .
$$

As showed above, $\|\phi\|_{\infty} \leq 1$. We have that $\left\|\phi(z) e_{0}\right\|^{2}=1$ to get

$$
\int_{\mathbb{T}^{2}}\left(|\phi(z)|^{2}-1\right)\left|e_{0}\right|^{2} d m_{2}=0
$$

Thus $|\phi(z)|=1$ almost all on the unit circle and so $\phi$ is an inner function. Lemma 24 gives that $\phi$ is a finite Blaschke product. This completes the proof.

\section{Structure of minimal Reducing subspaces}

In this section we will first show that every nontrivial minimal reducing subspace of $\phi(\mathcal{B})$ is orthogonal to the distinguished subspace $\mathcal{M}_{0}$ if it is other than $\mathcal{M}_{0}$. We will show the proof of Theorem 3 in the section.

Theorem 27. Suppose that $\Omega$ is a nontrivial minimal reducing subspace for $\phi(\mathcal{B})$. If $\Omega$ does not equal $\mathcal{M}_{0}$ then $\Omega$ is a subspace of $\mathcal{M}_{0}^{\perp}$. 
Proof. By Lemma 16, there is a function $e$ in $\Omega \cap L_{0}$ such that $e=\lambda e_{0}+e_{1}$ for some constant $\lambda$ and a function $e_{1}$ in $\mathcal{M}_{0}^{\perp} \cap L_{0}$. By Theorem 18

$$
p_{1}(\phi(z), \phi(w)) e+d_{e}^{1} \in \Omega .
$$

Here $d_{e}^{1}$ is the function constructed in Theorem 18. Let

$$
E=\phi(\mathcal{B})^{*}[\phi(\mathcal{B}) e]-\frac{1}{2} e .
$$

Since $p_{1}(\phi(z), \phi(w)) e_{0}$ is in $\mathcal{H}$, we obtain

$$
\phi(\mathcal{B}) e_{0}=\frac{p_{1}(\phi(z), \phi(w)) e_{0}}{2} .
$$

Simple calculations give

$$
\begin{aligned}
& \left.E=\phi(\mathcal{B})^{*}\left\{\phi(\mathcal{B})\left[\lambda e_{0}+e_{1}\right]\right]\right\}-\frac{1}{2}\left[\lambda e_{0}+e_{1}\right] \\
& =-\frac{1}{2} \phi(\mathcal{B})^{*} P_{\mathcal{H}} d_{e_{1}}^{1} .
\end{aligned}
$$

The sixth equality holds because that $p_{1}(\phi(z), \phi(w)) e_{1}+d_{e_{1}}^{1} \in \mathcal{H}$. The eighth equality follows from that $d_{e_{1}}^{1}$ is in $\mathcal{L}_{\phi}$. We claim that $E \neq 0$. If this is not true, we would have

$$
\frac{1}{2} \phi(\mathcal{B})^{*} P_{\mathcal{H}} d_{e_{1}}^{1}=0 .
$$

This gives that $P_{\mathcal{H}} d_{e_{1}}^{1}$ is in $L_{0}$. And hence

$$
0=\left\langle P_{\mathcal{H}} d_{e_{1}}^{1}, d_{e_{1}}^{1}\right\rangle=\left\|d_{e_{1}}^{1}\right\|^{2} .
$$

This gives that $d_{e_{1}}^{1}=0$. Thus we obtain that $p_{1}(\phi(z), \phi(w)) e_{1} \in \mathcal{H}$. By Theorem 20, we get that $e_{1}$ is linearly dependent on $e_{0}$. This contradicts that $e_{1} \in \mathcal{M}_{0}^{\perp}$. By Theorem $18, P_{\mathcal{H}} d_{e_{1}}^{1}$ is in $\mathcal{M}$ and so is $E=-\frac{1}{2} \phi(\mathcal{B})^{*} P_{\mathcal{H}} d_{e_{1}}^{1}$. This implies that $E$ is in $\Omega \cap \mathcal{M}_{0}^{\perp}$. We conclude that $\Omega \cap \mathcal{M}_{0}^{\perp}=\Omega$ since $\Omega$ is minimal to complete the proof.

Lemma 28. If $\mathcal{M}$ and $\mathcal{N}$ are two mutually orthogonal reducing subspaces of $\phi(\mathcal{B})$, then $\widetilde{\mathcal{M}}$ is orthogonal to $\tilde{\mathcal{N}}$.

Proof. Let $f=\sum_{l, k \geq 0} \phi(z)^{l} \phi(w)^{k} m_{l k}$ and $g=\sum_{l, k \geq 0} \phi(z)^{l} \phi(w)^{k} n_{l k}$ for finite numbers of elements $m_{l k} \in \mathcal{M}$ and $n_{l k} \in \mathcal{N}$. Then

$$
\begin{aligned}
\langle f, g\rangle & =\left\langle\sum_{l, k \geq 0} \phi(z)^{l} \phi(w)^{k} m_{l k}, \sum_{l, k \geq 0} \phi(z)^{l} \phi(w)^{k} n_{l k}\right\rangle \\
& =\sum_{l, k \geq 0} \sum_{l_{1}, k_{1} \geq 0}\left\langle\phi(z)^{l-l_{1}} \phi(w)^{k-k_{1}} m_{l k}, n_{l_{1} k_{1}}\right\rangle .
\end{aligned}
$$

Since $\mathcal{M}$ is orthogonal to $\mathcal{N}$ and both $\mathcal{M}$ and $\mathcal{N}$ are invariant subspaces of $T_{\phi(z)}^{*}$ and $T_{\phi(w)}^{*}$, the above inner product $\langle f, g\rangle$ must be zero. Thus we conclude that $\widetilde{\mathcal{M}}$ is orthogonal to $\widetilde{\mathcal{N}}$ to complete the proof.

Proof of Theorem 2. Suppose that $\left\{e_{1}^{(M)}, \cdots, e_{q_{M}}^{(M)}\right\}$ form a basis of $\mathcal{M} \cap L_{0}$. First we show

$$
\operatorname{span}\left\{e_{1}^{(M)}, \cdots, e_{q_{M}}^{(M)} ; d_{e_{1}^{(M)}}^{1}, \cdots, d_{e_{q_{M}}^{(M)}}^{1}\right\} \subset L_{\widetilde{\mathcal{M}}} .
$$

Note that $\left\{e_{1}^{(M)}, \cdots, e_{q_{M}}^{(M)} ; d_{e_{1}^{(M)}}^{1}, \cdots, d_{e_{q_{M}}^{(M)}}^{1}\right\}$ are contained in $\mathcal{L}_{\phi}$. It suffices to show

$$
\left\{e_{1}^{(M)}, \cdots, e_{q_{M}}^{(M)} ; d_{e_{1}^{(M)}}^{1}, \cdots, d_{e_{q_{M}}^{(M)}}^{1}\right\} \subset \widetilde{\mathcal{M}} .
$$


Since $\mathcal{M} \cap L_{0}$ contains $\left\{e_{1}^{(M)}, \cdots, e_{q_{M}}^{(M)}\right\}$, for each $l, k \geq 0, \phi(z)^{l} \phi^{k}(w) e_{i}^{(M)}$ is in $\widetilde{\mathcal{M}}$ for $1 \leq i \leq q_{M}$. Thus $p_{1}(\phi(z), \phi(w)) e_{i}^{(M)}$ is in $\widetilde{\mathcal{M}}$. By Theorem 18 , we have

$$
p_{1}(\phi(z), \phi(w)) e_{i}^{(M)}+d_{e_{i}^{(M)}}^{1} \in \mathcal{M}
$$

So we have that $d_{e_{i}^{(M)}}^{1} \in \widetilde{\mathcal{M}}$, to obtain

$$
\operatorname{span}\left\{e_{1}^{(M)}, \cdots, e_{q_{M}}^{(M)} ; d_{e_{1}^{(M)}}^{1}, \cdots, d_{e_{q_{M}}^{(M)}}^{1}\right\} \subset L_{\widetilde{\mathcal{M}}} .
$$

Next we will show that $\left\{e_{1}^{(M)}, \cdots, e_{q_{M}}^{(M)} ; d_{e_{1}^{(M)}}^{1}, \cdots, d_{e_{q_{M}}^{(M)}}^{1}\right\}$ are linearly independent. Suppose that for some constants $\lambda_{i}$ and $\mu_{i}$,

$$
\sum_{i=1}^{q} \lambda_{i} e_{i}^{(M)}+\sum_{i=1}^{q} \mu_{i} d_{e_{i}^{(M)}}^{1}=0 .
$$

Thus

$$
\sum_{i=1}^{q} \lambda_{i} e_{i}^{(M)}=-\sum_{i=1}^{q} \mu_{i} d_{e_{i}^{(M)}}^{1} .
$$

The right hand side of the above equality is in $L_{0}$ but the left hand side of the equality is orthogonal to $L_{0}$. So we have

$$
\sum_{i=1}^{q} \lambda_{i} e_{i}^{(M)}=0
$$

and

$$
\sum_{i=1}^{q} \mu_{i} d_{e_{i}^{(M)}}^{1}=0 .
$$

The first equality gives that $\lambda_{i}=0$ and the second equality gives

$$
d_{\sum_{i=1}^{q} \mu_{i} e_{i}^{(M)}}^{1}=0 .
$$

Because $\mathcal{M}$ is orthogonal to $\mathcal{M}_{0}$, by Theorem 20, we have

$$
\sum_{i=1}^{q} \mu_{i} e_{i}^{(M)}=0
$$

This gives that $\mu_{i}=0$. Hence $\left\{e_{1}^{(M)}, \cdots, e_{q_{M}}^{(M)} ; d_{e_{1}^{(M)}}^{1}, \cdots, d_{e_{q_{M}}^{(M)}}^{1}\right\}$ are linearly independent. So far, we have obtained

To finish the proof, we need only to show that

$$
\operatorname{dim} L_{\widetilde{\mathcal{M}}} \geq 2 q_{M} .
$$

$$
\operatorname{dim} L_{\widetilde{\mathcal{M}}} \leq 2 q_{M} .
$$

To do so, we consider the decomposition of $\mathcal{H}$,

$$
\mathcal{H}=\mathcal{M}_{0} \oplus \mathcal{M} \oplus\left[\mathcal{M}_{0}^{\perp} \cap \mathcal{M}^{\perp}\right]
$$

and

$$
L_{0}=\left[\mathcal{M}_{0} \cap L_{0}\right] \oplus\left[\mathcal{M} \cap L_{0}\right] \oplus\left\{\left[\mathcal{M}_{0}^{\perp} \cap \mathcal{M}^{\perp}\right] \cap L_{0}\right\}
$$

Then

$$
\begin{aligned}
\operatorname{dim}\left\{\left[\mathcal{M}_{0}^{\perp} \cap \mathcal{M}^{\perp}\right] \cap L_{0}\right\} & =\operatorname{dim} L_{0}-\operatorname{dim}\left[\mathcal{M}_{0} \cap L_{0}\right]-\operatorname{dim}\left[\mathcal{M} \cap L_{0}\right] \\
& =N-1-q_{M} .
\end{aligned}
$$

Letting $\mathcal{N}=\left[\mathcal{M}_{0}^{\perp} \cap \mathcal{M}^{\perp}\right]$, Lemma 28 gives

$$
\mathcal{K}_{\phi}=\widetilde{\mathcal{M}_{0}} \oplus \widetilde{\mathcal{M}} \oplus \widetilde{\mathcal{N}}
$$


and

Replacing $\mathcal{M}$ by $\mathcal{N}$ in the above argument gives

$$
\mathcal{L}_{\phi}=L_{\widetilde{\mathcal{M}}_{0}} \oplus L_{\widetilde{\mathcal{M}}} \oplus L_{\widetilde{\mathcal{N}}}
$$

$$
\operatorname{dim}_{\widetilde{\mathcal{N}}} \geq 2\left(N-1-q_{M}\right) .
$$

By Theorem 15, so we have

$$
2 N-1=1+\operatorname{dim}\left[L_{\widetilde{\mathcal{M}}}\right]+\operatorname{dim}\left[L_{\widetilde{\mathcal{N}}}\right] .
$$

Hence

$$
\begin{aligned}
\operatorname{dim}\left[L_{\widehat{\mathcal{M}}}\right] & =2 N-2-\operatorname{dim}\left[L_{\widehat{\mathcal{N}}}\right] \\
& \leq 2 N-2-2\left(N-1-q_{M}\right)=2 q_{M} .
\end{aligned}
$$

This completes the proof.

Lemma 29. Suppose that $\mathcal{M}, \mathcal{N}$, and $\Omega$ are three distinct nontrivial minimal reducing subspaces of $\phi(\mathcal{B})$ such that

$$
\Omega \subset \mathcal{M} \oplus \mathcal{N} .
$$

If $\mathcal{M}, \mathcal{N}$, and $\Omega$ are orthogonal to $\mathcal{M}_{0}$, then

$$
\widetilde{\mathcal{M}} \cap \widetilde{\Omega}=\widetilde{\mathcal{N}} \cap \widetilde{\Omega}=\{0\} .
$$

Proof. Since the intersection $\widetilde{\mathcal{M}} \cap \widetilde{\Omega}$ is also a reducing subspace of the pair of isometries $T_{\phi(z)}$ and $T_{\phi(w)}^{*}$, the Wold decomposition of the pair of isometries on $\widetilde{\mathcal{M}} \cap \widetilde{\Omega}$ gives

$$
\widetilde{\mathcal{M}} \cap \widetilde{\Omega}=\oplus_{l, k \geq 0} \phi(z)^{l} \phi(w)^{k} L_{\widetilde{\mathcal{M}} \cap \widetilde{\Omega}},
$$

where $L_{\widetilde{\mathcal{M}} \cap \tilde{\Omega}}$ is the wandering space given by

$$
\begin{aligned}
L_{\widetilde{\mathcal{M}} \cap \widetilde{\Omega}} & =\operatorname{ker}_{\phi(z)}^{*} \cap T_{\phi(w)}^{*} \cap \widetilde{\mathcal{M}} \cap \widetilde{\Omega} \\
& =\left[\operatorname{ker} T_{\phi(z)}^{*} \cap T_{\phi(w)}^{*} \cap \widetilde{\mathcal{M}}\right] \cap\left[\operatorname{ker} T_{\phi(z)}^{*} \cap T_{\phi(w)}^{*} \cap \widetilde{\Omega}\right] \\
& =L_{\widetilde{\mathcal{M}}} \cap L_{\widetilde{\Omega}} .
\end{aligned}
$$

To prove that $\widetilde{\mathcal{M}} \cap \widetilde{\Omega}=\{0\}$, it suffices to show

$$
L_{\widetilde{\mathcal{M}}} \cap L_{\widetilde{\Omega}}=\{0\} .
$$

To do this, let $q \in L_{\widetilde{\mathcal{M}}} \cap L_{\widetilde{\Omega}}$. By Theorem 2, there are functions $e_{M}, \tilde{e}_{M} \in \mathcal{M} \cap L_{0}$ and $e_{\Omega}, \tilde{e}_{\Omega} \in \Omega \cap L_{0}$ such that

$$
\begin{aligned}
q & =e_{M}+d_{\tilde{e}_{M}}^{1} \\
& =e_{\Omega}+d_{\tilde{e}_{\Omega}}^{1} .
\end{aligned}
$$

The above two equalities give

$$
e_{M}-e_{\Omega}=d_{\tilde{e}_{M}-\tilde{e}_{\Omega}}^{1} .
$$

On the other hand, $d_{\tilde{e}_{M}-\tilde{e}_{\Omega}}^{1}$ is orthogonal to $L_{0}$. Thus

$$
d_{\tilde{e}_{M}-\tilde{e}_{\Omega}}^{1}=e_{M}-e_{\Omega}=0 .
$$

This gives

$$
e_{M}=e_{\Omega}
$$

But $e_{M}$ is in $\mathcal{M}$ and $e_{\Omega}$ is in $\Omega$ and hence both $e_{M}$ and $e_{\Omega}$ are zero. Since $d_{\tilde{e}_{M}-\tilde{e}_{\Omega}}^{1}=0$, Theorem 20 implies that $\tilde{e}_{M}-\tilde{e}_{\Omega}$ linearly depends on $e_{0}$. Since both $\mathcal{M}$ and $\Omega$ are orthogonal to $\mathcal{M}_{0}$, we have that $\tilde{e}_{M}=\tilde{e}_{\Omega}$. Thus we obtain $\tilde{e}_{M}=0$ to conclude that $q=0$, as desired. So

$$
\widetilde{\mathcal{M}} \cap \widetilde{\Omega}=\{0\} .
$$


Similarly we obtain

$$
\widetilde{\mathcal{N}} \cap \widetilde{\Omega}=\{0\} .
$$

Lemma 30. Suppose that $\mathcal{M}, \mathcal{N}$, and $\Omega$ are three distinct nontrivial minimal reducing subspaces of $\phi(\mathcal{B})$ such that

$$
\Omega \subset \mathcal{M} \oplus \mathcal{N} \text {. }
$$

If $\mathcal{M}, \mathcal{N}$, and $\Omega$ are orthogonal to $\mathcal{M}_{0}$, then

$$
P_{\widetilde{\mathcal{M}}} L_{\widetilde{\Omega}}=L_{\widetilde{M}}
$$

and

$$
P_{\widetilde{\mathcal{N}}} L_{\widetilde{\Omega}}=L_{\widetilde{N}}
$$

where $P_{\widetilde{\mathcal{M}}}$ denotes the orthogonal projection from $H^{2}\left(\mathbb{T}^{2}\right)$ onto $\widetilde{\mathcal{M}}$.

Proof. Since $\mathcal{M}$ is orthogonal to $\mathcal{N}$, Lemma 28 gives that $\widetilde{\mathcal{M}}$ is orthogonal to $\widetilde{\mathcal{N}}$ and

$$
\widetilde{\Omega} \subset \widetilde{\mathcal{M}} \oplus \widetilde{\mathcal{N}} \text {. }
$$

We will show that $P_{\widetilde{\mathcal{M}}} L_{\widetilde{\Omega}}=L_{\widetilde{M}}$.

Since $\Omega \subset \mathcal{M} \oplus \mathcal{\mathcal { N }}$, we have

$$
\Omega \cap L_{0} \subset\left[\mathcal{M} \cap L_{0}\right] \oplus\left[\mathcal{N} \cap L_{0}\right] .
$$

For each $e^{(\Omega)} \in \Omega \cap L_{0}$, there are two functions $e^{(M)} \in \mathcal{M} \cap L_{0}$ and $e^{(N)} \in \mathcal{N} \cap L_{0}$ such that

$$
\begin{gathered}
e^{(\Omega)}=e^{(M)}+e^{(N)} \\
d_{e^{(\Omega)}}^{1}=d_{e^{(M)}}^{1}+d_{e^{(N)}}^{1} .
\end{gathered}
$$

By Theorem 2, $d_{e^{(M)}}^{1}$ is in $\widetilde{\mathcal{M}}$ and $d_{e^{(N)}}^{1}$ is in $\tilde{\mathcal{N}}$. Since $\mathcal{M}, \mathcal{N}$, and $\Omega$ are orthogonal to $\mathcal{M}_{0}$, the above decompositions are unique. Thus

$$
P_{\widetilde{\mathcal{M}}} e^{(\Omega)}=e^{(M)}
$$

and

$$
P_{\widetilde{\mathcal{M}}} d_{e^{(\Omega)}}^{1}=d_{e^{(M)}}^{1} .
$$

So for each $f=e^{(\Omega)}+d_{\tilde{e}^{(\Omega)}}^{1} \in L_{\tilde{\Omega}}$, where $e^{(\Omega)}$ and $\tilde{e}^{(\Omega)}$, we have

$$
P_{\widetilde{\mathcal{M}}} f=e^{(M)}+d_{\tilde{e}^{(M)}}^{1}
$$

is in $L_{\widetilde{\mathcal{M}}}$ to obtain

$$
P_{\widetilde{\mathcal{M}}} L_{\tilde{\Omega}} \subset L_{\widetilde{\mathcal{M}}}
$$

To prove that $P_{\widetilde{\mathcal{M}}} L_{\tilde{\Omega}}=L_{\widetilde{\mathcal{M}}}$, it suffices to show that

$$
P_{\widetilde{\mathcal{M}}}: L_{\tilde{\Omega}} \rightarrow L_{\widetilde{\mathcal{M}}}
$$

is subjective. If this is not so, by Theorem 2, there are two functions $e, \tilde{e} \in \mathcal{M} \cap L_{0}$ such that $0 \neq e+d_{\tilde{e}}^{1}$ is orthogonal to $P_{\widetilde{\mathcal{M}}} L_{\tilde{\Omega}}$.

Assume that $\left\{e_{1}, \cdots, e_{q_{\Omega}}\right\}$ are a basis of $\Omega \cap L_{0}$. Then

$$
P_{\widetilde{\mathcal{M}}} L_{\tilde{\Omega}}=\operatorname{span}\left\{e_{1}^{(M)}, \cdots, e_{q_{\Omega}}^{(M)} ; d_{e_{1}^{(M)}}^{1}, \cdots, d_{e_{q_{\Omega}}^{(M)}}^{1}\right\} .
$$

If $e \neq 0$, then $\left\langle e, e_{i}^{(M)}\right\rangle=0$, for $1 \leq i \leq q_{\Omega}$. Thus

$$
\begin{aligned}
0 & =\left\langle e, e_{i}^{(M)}\right\rangle \\
& =\left\langle e, e_{i}^{(M)}+e_{i}^{(N)}\right\rangle=\left\langle e, e_{i}\right\rangle,
\end{aligned}
$$

and

$$
\left\langle e, d_{e_{i}}^{1}\right\rangle=0,
$$


for each $1 \leq i \leq q_{\Omega}$. So $e$ is orthogonal to $L_{\tilde{\Omega}}=\operatorname{span}\left\{e_{1}, \cdots, e_{q_{\Omega}} ; d_{e_{1}}^{1}, \cdots, d_{e_{q_{\Omega}}}^{1}\right\}$. Noting $e$ is in $L_{0}$, we see that $e$ is orthogonal to $\phi(z)^{l} \phi(w)^{k} L_{\tilde{\Omega}}$, for each $l>0$ or $k>0$. This gives that $e$ is orthogonal to $\tilde{\Omega}$ and hence orthogonal to $\Omega$. Since $e$ is in $\mathcal{M}$, $e$ must be orthogonal to the closure of $P_{\mathcal{M}} \Omega \subset \mathcal{M}$, which is also a reducing subspace of $\phi(\mathcal{B})$. Therefore $e$ is orthogonal to $\mathcal{M}$, which is a contradiction.

If $e=0$, then $d_{\tilde{e}}^{1} \neq 0$ and

$$
\begin{aligned}
0 & =\left\langle d_{\tilde{e}}^{1}, d_{e_{i}^{(M)}}^{1}\right\rangle \\
& =\left\langle d_{\tilde{e}}^{1}, P_{\widetilde{\mathcal{M}}} d_{e_{i}}^{1}\right\rangle=\left\langle d_{\tilde{e}}^{1}, d_{e_{i}}^{1}\right\rangle,
\end{aligned}
$$

and

$$
\left\langle d_{\tilde{e}}^{1}, e_{i}\right\rangle=0,
$$

for each $1 \leq i \leq q_{\Omega}$. This gives that $d_{\tilde{e}}^{1}$ is orthogonal to $L_{\tilde{\Omega}}$. But $d_{\tilde{e}}^{1}$ is also in $\mathcal{L}_{\phi}$. We have that for any $f \in L_{\tilde{\Omega}}$,

$$
\left\langle d_{\tilde{e}}^{1}, \phi(z)^{l} \phi(w)^{k} f\right\rangle=0,
$$

for $l>0$ or $k>0$. We have that $d_{\tilde{e}}^{1}$ is orthogonal to $\tilde{\Omega}$ and hence orthogonal to $\Omega$ to obtain that $P_{\mathcal{H}} d_{\tilde{e}}^{1}$ is orthogonal to $\Omega$. On the other hand, by Theorem 18, $P_{\mathcal{H}} d_{\tilde{e}}^{1}$ is in $\mathcal{M}$. Thus $P_{\mathcal{H}} d_{\tilde{e}}^{1}$ is orthogonal to the closure of $P_{\mathcal{M}} \Omega$ and so $P_{\mathcal{H}} d_{\tilde{e}}^{1}$ must be zero because the closure of $P_{\mathcal{M}} \Omega$ equals $\mathcal{M}$. Therefore,

$$
\begin{aligned}
0 & =\left\langle P_{\mathcal{H}} d_{\tilde{e}}^{1}, p_{1}(\phi(z), \phi(w)) \tilde{e}+d_{\tilde{e}}^{1}\right\rangle \\
& =\left\langle d_{\tilde{e}}^{1}, p_{1}(\phi(z), \phi(w)) \tilde{e}+d_{\tilde{e}}^{1}\right\rangle \\
& =\left\langle d_{\tilde{e}}^{1}, d_{\tilde{e}}^{1}\right\rangle=\left\|d_{\tilde{e}}^{1}\right\|^{2} .
\end{aligned}
$$

The second equality follows from that $p_{1}(\phi(z), \phi(w)) \tilde{e}+d_{\tilde{e}}^{1}$ is in $\mathcal{H}$ and the third equality follows that $d_{\tilde{e}}^{1}$ is orthogonal to $p_{1}(\phi(z), \phi(w)) \tilde{e}$. This gives that $d_{\tilde{e}}^{1}=0$, which is a contradiction. We have obtained that $P_{\widetilde{\mathcal{M}}}: L_{\tilde{\Omega}} \rightarrow L_{\widetilde{\mathcal{M}}}$ is subjective and hence

$$
P_{\widetilde{\mathcal{M}}} L_{\tilde{\Omega}}=L_{\widetilde{\mathcal{M}}}
$$

Similarly we obtain

$$
P_{\widetilde{\mathcal{N}}} L_{\tilde{\Omega}}=L_{\widetilde{\mathcal{N}}}
$$

This completes the proof.

Now we are ready to prove Theorem 3 .

Proof of Theorem 3. First we will show

$$
P_{\mathcal{M}}=P_{\mathcal{H}} P_{\widetilde{\mathcal{M}}}
$$

Let $\mathcal{N}_{1}$ denote the orthogonal complementary of $\mathcal{M} \oplus \mathcal{N}$ in $\mathcal{H}$. Write

$$
\mathcal{H}=\mathcal{M} \oplus \mathcal{N} \oplus \mathcal{N}_{1}
$$

Lemma 28 gives

$$
\widetilde{\mathcal{H}}=\widetilde{\mathcal{M}} \oplus \widetilde{\mathcal{N}} \oplus \widetilde{\mathcal{N}_{1}} .
$$

For each function $f$ in $H^{2}\left(\mathbb{T}^{2}\right)$, write

$$
\begin{aligned}
f & =f_{\widetilde{\mathcal{H}}} \oplus f_{2} \\
& =f_{\widetilde{\mathcal{M}}^{\mathcal{M}}} \oplus f_{\widetilde{\mathcal{N}}} \oplus f_{\mathcal{\mathcal { N }}_{1}} \oplus f_{2},
\end{aligned}
$$

where $f_{2}$ is orthogonal to $\widetilde{\mathcal{H}}, f_{\widetilde{\mathcal{H}}} \in \widetilde{\mathcal{H}}, f_{\widetilde{\mathcal{M}}} \in \widetilde{\mathcal{M}}, f_{\widetilde{\mathcal{N}}} \in \widetilde{\mathcal{N}}$, and $f_{\widetilde{\mathcal{N}}_{1}} \in \widetilde{\mathcal{N}_{1}}$. Since $\widetilde{\mathcal{M}}$ contains $\mathcal{M}$, we write

$$
f_{\widetilde{\mathcal{M}}}=f_{\mathcal{M}} \oplus f_{3}
$$


for two functions $f_{\mathcal{M}} \in \mathcal{M}$ and $f_{3} \in \widetilde{\mathcal{M}} \ominus \mathcal{M}$. Thus $f_{3}$ is orthogonal to both $\widetilde{\mathcal{N}}$ and $\widetilde{\mathcal{N}_{1}}$ and hence orthogonal to both $\mathcal{N}$ and $\mathcal{N}_{1}$. So $f_{3}$ is orthogonal to

$$
\mathcal{H}=\mathcal{M} \oplus \mathcal{N} \oplus \mathcal{N}_{1}
$$

This gives that $P_{\mathcal{H}} f_{3}=0$. We have

$$
\begin{aligned}
P_{\mathcal{H}} P_{\widetilde{\mathcal{M}}}^{f} & =P_{\mathcal{H}} f_{\widetilde{\mathcal{M}}} \\
& =P_{\mathcal{H}^{f}} f_{\mathcal{M}}+P_{\mathcal{H}} f_{3} \\
& =P_{\mathcal{H}}^{f} f_{\mathcal{M}}=f_{\mathcal{M}},
\end{aligned}
$$

and

to get

$$
P_{\mathcal{M}}^{f}=f_{\mathcal{M}}
$$

$$
P_{\mathcal{M}}=P_{\mathcal{H}} P_{\widetilde{\mathcal{M}}}
$$

Next we will show that $P_{\mathcal{M}}$ is subjective from $\Omega$ onto $\mathcal{M}$. For each $q \in \mathcal{M}$, by Lemma 30, there are functions $q_{l k} \in L_{\tilde{\Omega}}$ such that

$$
q=\sum_{l, k \geq 0} \phi(z)^{l} \phi(w)^{k} m_{l k}
$$

and

$$
\|q\|^{2}=\sum_{l, k \geq 0}\left\|m_{l k}\right\|^{2}<\infty,
$$

where $m_{l k}=P_{\mathcal{M}^{q k}}$. Since $L_{\tilde{\Omega}}$ and $L_{\widetilde{M}}$ are finite dimension spaces, there are two positive constants $c_{1}$ and $c_{2}$ such that

$$
c_{1}\left\|q_{l k}\right\| \leq\left\|m_{l k}\right\| \leq c_{2}\left\|q_{l k}\right\| .
$$

Define

$$
\tilde{q}=\sum_{l, k \geq 0} \phi(z)^{k} \phi(w)^{l} q_{l k}
$$

Thus

$$
\begin{aligned}
\|\tilde{q}\|^{2} & =\sum_{l, k \geq 0}\left\|q_{l k}\right\|^{2} \\
& \leq c_{2} \sum_{l, k \geq 0}\left\|m_{l k}\right\|^{2}<\infty .
\end{aligned}
$$

So we obtain that $\tilde{q}$ is in $\tilde{\Omega}$, and

$$
\begin{aligned}
\tilde{q} & =\sum_{l, k \geq 0} \phi(z)^{l} \phi(w)^{k} q_{l k} \\
& =\sum_{l, k \geq 0} \phi(z)^{l} \phi(w)^{k}\left[P_{\mathcal{\mathcal { M }}^{l k}}+P_{\left.\mathcal{\mathcal { N }}^{q_{l k}}\right]}\right. \\
& =\sum_{l, k \geq 0} \phi(z)^{l} \phi(w)^{k} m_{l k}+\sum_{l, k \geq 0} \phi(z)^{l} \phi(w)^{k}\left[P_{\mathcal{\mathcal { N }}^{q l k}}\right] \\
& =q+q_{N},
\end{aligned}
$$

where $q_{N}=\sum_{l, k \geq 0} \phi(z)^{k} \phi(w)^{l}\left[P_{\widetilde{\mathcal{N}}^{q l k}}\right]$ is in $\widetilde{N}$. Hence $P_{\widetilde{\mathcal{M}}} \tilde{q}=q$. We have

$$
P_{\mathcal{H}^{P}} P_{\overline{\mathcal{M}}} \tilde{q}=P_{\mathcal{H}^{q}}=q,
$$

to obtain

$$
P_{\mathcal{M}} \tilde{q}=P_{\mathcal{H}} P_{\widetilde{\mathcal{M}}^{q}=q}
$$


Since $\mathcal{M}$ is a subspace of $\mathcal{H}, P_{\mathcal{M}}=P_{\mathcal{M}} P_{\mathcal{H}}$. Thus

$$
P_{\mathcal{M}} P_{\mathcal{H}} \tilde{q}=P_{\mathcal{M}} \tilde{q}=q .
$$

Writing $q_{l k}=e_{k l}^{(\Omega)}+d_{\tilde{e}_{k l}^{(\Omega)}}^{1}$ for functions $e_{k l}^{(\Omega)}, \tilde{e}_{k l}^{(\Omega)} \in \Omega \cap L_{0}$, we have

$$
\begin{aligned}
P_{\mathcal{H}} \tilde{q} & =\sum_{l, k \geq 0} P_{\mathcal{H}}\left(\phi(z)^{l} \phi(w)^{k} q_{l k}\right) \\
& =\sum_{l, k \geq 0} P_{\mathcal{H}} \phi(z)^{l} \phi(w)^{k}\left(e_{k l}^{(\Omega)}+d_{\tilde{e}_{k l}^{(\Omega)}}^{1}\right) \\
& =\sum_{l, k \geq 0}\left(P_{\mathcal{H}} \phi(z)^{l} \phi(w)^{k} e_{k l}^{(\Omega)}\right)+\sum_{l, k \geq 0}\left(P_{\mathcal{H}} \phi(z)^{l} \phi(w)^{k} d_{\tilde{e}_{k l}^{(\Omega)}}^{1}\right) \\
& =\sum_{l, k \geq 0}\left(P_{\mathcal{H} \phi(z)^{l} \phi(w)^{k}} e_{k l}^{(\Omega)}\right)+\sum_{l, k \geq 0}\left[P_{\mathcal{H}} \phi(z)^{l} \phi(w)^{k}\left(P_{\mathcal{H}} d_{\tilde{e}_{k l}^{(\Omega)}}^{1}\right)\right]
\end{aligned}
$$

The last equality follows from that $\phi(z)^{l} \phi(w)^{k}\left(1-P_{\mathcal{H}}\right) d_{\tilde{e}_{k l}^{(\Omega)}}^{1}$ is orthogonal to $\mathcal{H}$. The the first sum in the last equality is in $\Omega$ and Theorem 18 gives that the second sum in the equality is in $\Omega$ also. Letting $\omega=P_{\mathcal{H}} \tilde{q}$, we have proved that $P_{\mathcal{M}} \omega=q$ to get that

$$
P_{\mathcal{M}} \Omega=\mathcal{M} \text {. }
$$

On the other hand, $\operatorname{ker}\left[\left.P_{\mathcal{M}}\right|_{\Omega}\right] \subset \Omega$ is a reducing subspace of $\phi(\mathcal{B})$. Since $\Omega$ is a nontrivial minimal reducing spaces of $\phi(\mathcal{B})$, we see that $\operatorname{ker}\left[\left.P_{\mathcal{M}}\right|_{\Omega}\right]=\{0\}$. This implies that $P_{\mathcal{M}}: \Omega \rightarrow \mathcal{M}$ is bijective and bounded. By the closed graph theorem we conclude that $\left.P_{\mathcal{M}}\right|_{\Omega}$ is invertible.

Similarly we can show that that $P_{\mathcal{N}} \mid \Omega$ is invertible. Define

$$
S=\left[P_{\mathcal{N}} \mid \Omega\right]\left[\left.P_{\mathcal{M}}\right|_{\Omega}\right]^{-1} .
$$

Then $S$ is an invertible operator from $\mathcal{M}$ onto $\mathcal{N}$. Both $S$ and $S^{*}$ commute with $\phi(\mathcal{B})$ because $\Omega, \mathcal{M}$ and $\mathcal{N}$ are three distinct nontrivial minimal reducing subspaces for $\phi(\mathcal{B})$. Thus $S^{*} S$ commutes with $\phi(\mathcal{B})$. Making the polar decomposition of $S$, we write

$$
S=U|S|,
$$

for some unitary operator $U$ from $\mathcal{M}$ onto $\mathcal{N}$, where $|S|=\left[S^{*} S\right]^{1 / 2}$. So $U$ commutes with both $\phi(\mathcal{B})$ and $\phi(\mathcal{B})^{*}$. This completes the proof.

Theorem 31. Let $\mathcal{M}$ and $\mathcal{N}$ be two distinct nontrivial minimal reducing subspaces of $\phi(\mathcal{B})$. Then either they are orthogonal or $\phi(\mathcal{B})$ has two distinct unitarily equivalent reducing subspaces and has also infinitely many minimal reducing subspaces.

Proof. Let $\mathcal{M}$ and $\mathcal{N}$ be two distinct nontrivial minimal reducing subspaces of $\phi(\mathcal{B})$. Consider

$$
\mathcal{W}=[\operatorname{closure}(\boldsymbol{M}+\mathcal{N})] \ominus \mathcal{M} .
$$

Then $\mathcal{W}$ is a reducing subspace of $\phi(\mathcal{B})$. For each $y \in \operatorname{closure}(\mathcal{M}+\mathcal{N})$, we have

$$
y=P_{\mathcal{M}} y+P_{\mathcal{M}^{\perp}} y .
$$

Thus

$$
\text { closure }(\mathcal{M}+\mathcal{N})=\mathcal{M} \oplus \mathcal{W} .
$$

If $\mathcal{M}$ and $\mathcal{N}$ are not orthogonal, by Theorem $27, \mathcal{M}, \mathcal{N}$ are orthogonal to the distigushied minimal reducing subspace $\mathcal{M}_{0}$, and then $\mathcal{N}$ does not equal $\mathcal{W}$ and

$$
\mathcal{N} \subset \mathcal{M} \oplus \mathcal{W} .
$$

Now we show that $\mathcal{W}$ is a minimal reducing subspace of $\phi(\mathcal{B})$. Since $\mathcal{M}$ and $\mathcal{N}$ are distinct and they are minimal reducing subspaces, we have that the intersection of $\mathcal{M}$ and $\mathcal{N}$ equals 0. 
Noting that $\mathcal{N} \cap \mathcal{M}^{\perp}$ is a reducing subspace and contained in $\mathcal{N}$, we see that $\mathcal{N} \cap \mathcal{M}^{\perp}$ equals 0 to get that

$$
\operatorname{ker}\left(P_{\mathcal{M}} \mid \mathcal{N}\right)=\{0\} .
$$

This gives that for each $q \in \mathcal{N}, P_{\mathcal{M}} q \neq 0$. Since $\mathcal{N}$ is a minimal reducing subspace, for each $0 \neq q_{0}$ in $\mathcal{N}$, the closure of $\left\{q-P_{\mathcal{M}} q: q \in \mathcal{N}\right\}$ is the reducing subspace generated by $q_{0}-P_{\mathcal{M}} q_{0}$ which equals $\mathcal{W}$. Thus $\mathcal{W}$ is a minimal reducing subspace. By (6), we observe that $\mathcal{M}, \mathcal{N}$ and $\mathcal{W}$ satisfy the conditions in Theorem 3 . So $\mathcal{M}$ is unitarily equivalent to $\mathcal{W}$.

Now for each $\alpha$ in $[0,1]$, define

$$
\mathcal{N}_{\alpha}=\text { closure }\left\{q-\alpha P_{\mathcal{M}} q: q \in \mathcal{N}\right\} .
$$

As $\mathcal{N}$ is a minimal reducing subspace, each $\mathcal{N}_{\alpha}$ is a minimal reducing subspace. For $\alpha$ and $\beta$ in $[0,1]$, and $q_{1}$ and $q_{2}$ in $\mathcal{N}$, if

$$
q_{1}-\alpha P_{\mathcal{M}^{1}}^{q_{1}}=q_{2}-\beta P_{\mathcal{M}} q_{2},
$$

then

$$
q_{1}-q_{2}=\alpha P_{\mathcal{M}^{q_{1}}}-\beta P_{\mathcal{M}} q_{2} .
$$

The right hand side of the above equality is in $\mathcal{N}$ but the left hand side is in $\mathcal{M}$. Thus $q_{1}$ equals $q_{2}$ and $\alpha$ equals $\beta$. So $\mathcal{N}_{\alpha}$ does not equal $\mathcal{N}_{\beta}$ provided $\beta$ does not equal $\alpha$. Hence we get infinitely many minimal reducing subspaces to complete the proof.

Theorem 32. Let $\phi$ be a Blaschke product of finite order $N$. Then either $\phi(\mathcal{B})$ has infinitely many minimal reducing subspaces or the number of nontrivial reducing subspaces of $\phi(\mathcal{B})$ is less than or equal to $N$.

Proof. If $\phi(\mathcal{B})$ does not have infinitely many nontrivial reducing subspaces, by Theorem 31, any two distinct reducing subspaces must be orthogonal. Let $\left\{\mathcal{M}_{j}\right\}_{j=1}^{N_{1}}$ be the set of distinct minimal reducing subspaces of $\phi(\mathcal{B})$. Thus

$$
\oplus_{j=1}^{N_{1}} \mathcal{M}_{j} \subset \mathcal{H} .
$$

Lemma 16 gives that the dimension of $\mathcal{M}_{j} \cap L_{0}$ is at less one. So

$$
\operatorname{dim} L_{0} \geq \operatorname{dim}\left\{\left[\oplus_{j=1}^{N_{1}} \mathcal{M}_{j}\right] \cap L_{0}\right\} \geq N_{1} .
$$

On the other hand,

$$
L_{0}=\operatorname{ker} T_{\phi(z)}^{*} \cap \operatorname{ker} T_{\phi(w)}^{*} \cap \mathcal{H} .
$$

As pointed out before, the dimension of $L_{0}$ equals $N$. Thus

$$
N \geq N_{1} \text {. }
$$

So the number of nontrivial minimal reducing subspaces of $\phi(\mathcal{B})$ is less than or equal to the order $N$ of $\phi$. The proof is completed.

\section{Proofs of Theorems 4 AND 5}

In this section we will prove Theorems 4 and 5. For the Blaschke product $\phi(z)=z^{2} \frac{z-\alpha}{1-\bar{\alpha} z}$ with a nonzero point $\alpha$ in $\mathbb{D}$, for each $e$ in the wandering subspace of a reducing subspace of $\phi(\mathcal{B})$ we will be able to calculate $d_{e}^{0}$ in Theorem 21 and $L_{0}$ precisely. By Theorem 1, the fact that $d_{e}^{1}$ is orthogonal to $L_{0}$ leads to some algebraic equations. By solving the algebraic equations, we will show that $\phi(\mathcal{B})$ has only two nontrivial minimal reducing subspaces.

Theorem 33. For the Blaschke product $\phi(z)=z^{2} \frac{z-\alpha}{1-\bar{\alpha} z}$ with a nonzero point $\alpha$ in $\mathbb{D}, \phi(\mathcal{B})$ has only two minimal reducing subspaces $\left\{\mathcal{M}_{0}, \mathcal{M}_{0}^{\perp}\right\}$. 
Proof. For a given nonzero point $\alpha$ in the unit disk, let $\phi_{0}(z)=z \frac{z-\alpha}{1-\bar{\alpha} z}$. The Mittag-Leffler expansion of the finite Blaschke product $\phi_{0}$ is

$$
\phi_{0}(z)=-\frac{1-|\alpha|^{2}}{\bar{\alpha}^{2}}-\frac{1}{\bar{\alpha}} z+\frac{1-|\alpha|^{2}}{\bar{\alpha}^{2}} k_{\alpha}(z) .
$$

So

$$
\phi(z)=z \phi_{0}(z)=-\frac{1-|\alpha|^{2}}{\bar{\alpha}^{2}} z-\frac{1}{\bar{\alpha}} z^{2}+\frac{1-|\alpha|^{2}}{\bar{\alpha}^{2}} k_{\alpha}(z) .
$$

Hence

$$
e_{0}(z, w)=-\frac{1-|\alpha|^{2}}{\bar{\alpha}^{2}}-\frac{1}{\bar{\alpha}} p_{1}(z, w)+\frac{1-|\alpha|^{2}}{\bar{\alpha}^{2}} k_{\alpha}(z) k_{\alpha}(w) .
$$

It is easy to see that

$$
L_{0}=\operatorname{span}\left\{1, p_{1}(z, w), k_{\alpha}(z) k_{\alpha}(w)\right\}
$$

Theorem 25 gives

$$
\mathcal{M}_{0}=\operatorname{span}_{l \geq 0}\left\{p_{l}(\phi(z), \phi(w)) e_{0}\right\} .
$$

By Theorem 27, for each minimal reducing subspace $\Omega$ not equal to $\mathcal{M}_{0}, \Omega$ is a subspace of $\mathcal{M}_{0}^{\perp}$. So we need only to show that $\mathcal{M}_{0}^{\perp}$ is a minimal reducing subspace for $\phi(\mathcal{B})$.

Assume that $\mathcal{M}_{0}^{\perp}$ is not a minimal reducing subspace for $\phi(\mathcal{B})$. Then $\mathcal{H}$ is the direct sum of three reducing subspaces of $\phi(\mathcal{B})$. We may assume

$$
\mathcal{H}=\oplus_{i=0}^{2} \mathcal{M}_{i}
$$

Now choose a nonzero vector $e_{i}$ in the wandering subspace for $\mathcal{M}_{i}$, which is contained in $L_{0}$. Since $\left\{e_{i}\right\}_{i=0}^{2}$ are mutually orthogonal to each other, they form a basis for $L_{0}$. On the other hand, those functions $1, p_{1}(z, w)$ and $k_{\alpha}(z) k_{\alpha}(w)$ are a basis for $L_{0}$. Thus there are some constants $c_{i j}$ such that

$$
e_{i}=c_{i 0}+c_{i 1} p_{1}(z, w)+c_{i 2} k_{\alpha}(z) k_{\alpha}(w) .
$$

First we show that neither $c_{12}$ nor $c_{22}$ equals zero. Since

$$
\left\langle e_{0}, 1\right\rangle=e_{0}(0,0)=\phi_{0}(0)=0
$$

and

$$
\begin{aligned}
\left\langle e_{0}, p_{1}\right\rangle & =\left\langle e_{0}, p_{1}(z, z)\right\rangle=\left\langle e_{0}(z, 0), 2 z\right\rangle \\
& =2\left\langle\phi_{0}, z\right\rangle=-2 \alpha
\end{aligned}
$$

we have that 1 is in $\mathcal{M}_{0}^{\perp}$ but $p_{1}(z, w)$ is not in $\mathcal{M}_{0}^{\perp}$, to get that $c_{i 2} \neq 0$ for $i=1,2$.

Next we show that $e_{i}(0, \alpha)$ equals 0 for $i=0,1,2$. To do this, note that the dimension of the wandering subspace for each $\mathcal{M}_{i}$ equals 1 . By Theorem 1, there are constants $\beta_{i}$ and $\lambda_{i}$ such that

Thus for $i, j \geq 1$ and $i \neq j$,

$$
d_{e_{i}}^{1}=d_{e_{i}}^{0}+\beta_{i} e_{i}+\lambda_{i} e_{0}
$$

$$
\begin{aligned}
\left\langle d_{e_{i}}^{0}, e_{j}\right\rangle & =\left\langle d_{e_{i}}^{1}-\beta_{i} e_{i}-\lambda_{i} e_{0}, e_{j}\right\rangle \\
& =\left\langle d_{e_{i}}^{1}, e_{j}\right\rangle-\beta_{i}\left\langle e_{i}, e_{j}\right\rangle-\lambda_{i}\left\langle e_{0}, e_{j}\right\rangle=0 .
\end{aligned}
$$

The last equality follows from the fact that $d_{e_{i}}^{1}$ is orthogonal to $L_{0}$ and $\left\{e_{i}\right\}$ are an orthogonal basis for $L_{0}$.

By Theorem 21, we have

$$
\begin{aligned}
\left\langle d_{e_{i}}^{0}, e_{j}\right\rangle & =\left\langle w e_{i}(0, w) e_{0}(z, w)-\phi(w) e_{i}(z, w), e_{j}\right\rangle \\
& =\left\langle w e_{i}(0, w) e_{0}(z, w), e_{j}(z, w)\right\rangle
\end{aligned}
$$


Simple calculations give

$$
\begin{aligned}
\left\langle w e_{i}(0, w) e_{0}(z, w), p_{1}(z, w)\right\rangle & \left.=\left\langle w e_{i}(0, w) e_{0}(w, w), p_{1}(z, w)\right\rangle \text { (by Lemma } 7\right) \\
& =\left\langle w e_{i}(0, w) \phi^{\prime}(w), p_{1}(0, w)\right\rangle \text { (by Theorem 9) } \\
& =\left\langle w e_{i}(0, w) \phi^{\prime}(w), w\right\rangle=e_{i}(0,0) \phi^{\prime}(0)=0 .
\end{aligned}
$$

It is easy to see

$$
\left\langle w e_{i}(0, w) e_{0}(z, w), 1\right\rangle=0
$$

These give

$$
\begin{aligned}
\left\langle d_{e_{i}}^{0}, e_{j}\right\rangle & =c_{j 2}\left\langle w e_{i}(0, w) e_{0}(z, w), k_{\alpha}(z) k_{\alpha}(w)\right\rangle \\
& =c_{j 2}\left\langle w e_{i}(0, w) e_{0}(w, w), k_{\alpha}(z) k_{\alpha}(w)\right\rangle \\
& =c_{j 2}\left\langle w e_{i}(0, w) e_{0}(w, w), k_{\alpha}(w)\right\rangle \\
& =c_{j 2} \alpha e_{i}(0, \alpha) e_{0}(\alpha, \alpha) .
\end{aligned}
$$

Noting that $e_{0}(\alpha, \alpha)=\phi^{\prime}(\alpha) \neq 0$, we have $c_{j 2} e_{i}(0, \alpha)=0$, to get that $e_{i}(0, \alpha)=0$ for $i=1,2$. Also we have $e_{0}(0, \alpha)=\phi_{0}(\alpha)=0$. Since $\left\{e_{0}, e_{1}, e_{2}\right\}$ forms a basis for $L_{0}$ and $p_{1}(z, w)$ is in $L_{0}, p_{1}(z, w)$ is a linear combination of functions $e_{0}, e_{1}$ and $e_{2}$. Thus $p(0, \alpha)$ must be zero. But $p_{1}(0, \alpha)=\alpha \neq 0$. This leads to a contradiction. So $\mathcal{M}_{0}^{\perp}$ is a minimal reducing subspace of $\phi(\mathcal{B})$ to complete the proof

Now we are ready to prove Theorems 4 and 5 .

Proof of Theorem 4. Suppose that $\phi$ is a Blaschke product with three zeros. As pointed out in the first section $M_{\phi}$ is unitarily equivalent to $\phi(\mathcal{B})$, in the rest proof we will concern only $\phi(\mathcal{B})$.

First observe that for $\lambda \in \mathbb{D}$ and a subspace $\mathcal{M}$ of $\mathcal{H}, \mathcal{M}$ is a reducing subspace of $\phi(\mathcal{B})$ if and only if $\mathcal{M}$ is a reducing subspace of $\phi_{\lambda} \circ \phi(\mathcal{B})$.

If $\phi(z)$ has a multiple critical point in the unit disk, then

$$
\phi=\phi_{\lambda} \circ z^{3} \circ \phi_{\mu}
$$

for two numbers $\lambda, \mu \in \mathbb{D}$. Thus every reducing subspace of $\phi(\mathcal{B})$ is also a reducing subspace of $\phi_{\mu}(\boldsymbol{B})^{3}$. But $\phi_{\mu}(\boldsymbol{B})^{3}$ is unitarily equivalent to the direct sum of three weighted shifts and hence it has only three minimal reducing subspaces.

If $\phi$ does not have any multiple critical point in the unit disk, by Bochner's theorem [35], $\phi(z)$ always has a critical point $c$ in the unit disk. Let $\lambda=\phi(c)$. Then

$$
\phi_{\lambda} \circ \phi \circ \phi_{c}(z)=z^{2} \frac{z-a}{z-\bar{a} z},
$$

for some nonzero point $a \in \mathbb{D}$. Let $\psi(z)=\phi_{\lambda} \circ \phi \circ \phi_{c}(z)$. By Theorem 33, we conclude that $\psi(\mathcal{B})$ has only two minimal reducing subspaces. Hence $\phi(\mathcal{B})$ has only two minimal reducing subspaces. This completes the proof.

Proof of Theorem 5. Let $\phi$ be a Blaschke product with three zeros.

As in the proof of Theorem 4 , If $\phi(z)$ has a multiple critical point in the unit disk, then

$$
\phi=\phi_{\lambda} \circ z^{3} \circ \phi_{\mu}
$$

for two numbers $\lambda, \mu \in \mathbb{D}$. In this case, the Riemann surface of $\phi^{-1} \circ \phi$ over $\mathbb{D}$ has the same number of connected components as the one of $z^{-3} \circ z^{3}$ over $\mathbb{D}$ does. But the latter one has three connected components and $M_{\phi}$ has the only three nontrivial minimal reducing subspaces. Thus the number of nontrivial minimal reducing subspaces of $M_{\phi}$ equals the number of connected components of the Riemann surface of $\phi^{-1} \circ \phi$ over $\mathbb{D}$.

If $\phi$ does not have any multiple critical point in the unit disk, as in the proof of of Theorem $4, \phi(z)$ always has a critical point $c$ in the unit disk. Let $\lambda=\phi(c)$. Then

$$
\phi_{\lambda} \circ \phi \circ \phi_{c}(z)=\psi(z)
$$


where $\psi(z)=z^{2} \frac{z-a}{z-\bar{a} z}$ for some nonzero point $a \in \mathbb{D}$. By the example in [24], except for the trivial branch $z$, nontrivial branches of $\psi^{-1} \circ \psi$ are all continuations of one another. Thus the Riemann surface of $\psi^{-1} \circ \psi$ over $\mathbb{D}$ has only two connected components. So does the Riemann surface of $\phi^{-1} \circ \phi$ over $\mathbb{D}$. By Theorem 33, the number of nontrivial minimal reducing subspaces of $M_{\phi}$ equals the number of connected components of the Riemann surface of $\phi^{-1} \circ \phi$ over $\mathbb{D}$. This completes the proof.

\section{REFERENCES}

[1] A. Aleman, S. Richter and C. Sundberg, Beurling's theorem for the Bergman space, Acta Math., 177 (1996), no. $2,275-310$.

[2] I. Baker, J. Deddens and J. Ullman, A theorem on entire functions with applications to Toeplitz operators, Duke Math. J., 41(1974), 739-745.

[3] J. Ball, Hardy space expectation operators and reducing subspaces, Proc. Amer. Math. Soc., 47(1975), 351-357.

[4] H. Bercovici, C. Foias and C. Pearcy, Dual algebra with applications to invariant subspace and dilation theory, CBMS, Vol. 56.

[5] A. Brown, On a class of operators, Proc. Amer. Math. Soc., 4 (1953) 723-728.

[6] C. Cowen, The commutant of an analytic Toeplitz operator, Trans. Amer. Math. Soc., 239(1978), 1-31.

[7] M. Cowen and R. Douglas, Complex geometry and operator theory, Acta Math, 141 (1978), 187-261.

[8] R. Curto, Fredholm and invertible $n$-tuples of operators, the deformation problem, Trans. Amer. Math. Soc., 266(1981), 129-159.

[9] R. Douglas, Banach Algebra Techniques in Operator Theory, Academic press, New York and London, 1972.

[10] S. Ferguson and R. Rochberg, Higher order Hilbert-Schmidt Hankel forms and tensors of analytic kernels, Math. Scand., 96 (2005), no. 1, 117-146.

[11] S. Ferguson and R. Rochberg, Description of certain quotient Hilbert modules, Operator theory, 20, 93-109, Theta Ser. Adv. Math., 6, Theta, Bucharest, 2006.

[12] R. Douglas and V. Paulsen, Hilbert Modules over function algebras, Pitman Research Notes in Mathematics, Series 217, Lonman Group UK Limited, 1989.

[13] R. Douglas, R. Yang, Operator theory in the Hardy space over the bidisk I, Integral Equations Operator Theory 38 (2000), 207-221.

[14] J. Garnett, Bounded Analytic Functions, Academic Press Inc., 1981.

[15] P. Duren and A. Schuster, Bergman spaces., Mathematical Surveys and Monographs, 100. American Mathematical Society, Providence, RI, 2004.

[16] P. Halmos, Shifts on Hilbert spaces, J. Reine. Angew. Math., 208(1961) 102-112.

[17] H. Hedenmalm, B. Korenblum and K. Zhu, Theory of Bergman spaces, Graduate Texts in Mathematics, 199. Springer-Verlag, New York, 2000.

[18] J. Hu, S. Sun, X. Xu and D. Yu, Reducing subspace of analytic Toeplitz operators on the Bergman space, Integral equations operator theory, 49(2004), 387-395.

[19] B. Sz-Nagy and C. Foias, Harmonic Analysis of Operators on Hilbert Space, North-Holland, Amsterdam, 1970.

[20] E. Nordgren, Reducing subspaces of analytic Toeplitz operators, Duke Math. J., 34(1967), 175-181.

[21] S. Richter, Unitary equivalence of invariant subspaces of Bergman and Direchlet spaces, Pacific J. Math, 133(1988),151-156.

[22] S. Richter, On invariant subspaces of multiplication operators on Banach spaces of analytic functions, $\mathrm{Ph}$. Dissertation, Univ. of Michigan, 1986.

[23] W. Rudin, Function theory in polydiscs. W. A. Benjamin, Inc., New York-Amsterdam 1969 vii+188 pp.

[24] K. Stephenson, Analytic functions of finite valence, with applications to Toeplitz operators, Michigan Math. J., 32(1985), 5-19.

[25] K. Stephenson, Analytic functions and hypergroups of function pairs, Indiana Univ. Math. J., 31 (1982), 843-884.

[26] K. Stephenson, Analytic functions sharing level curves and tracts, Ann. of Math., (2) 123 (1986), no. 1, 107-144.

[27] M. Stessin and K. Zhu, Generalized factorization in Hardy spaces and the commutant of Toeplitz operators, Canad. J. Math., 55 (2003), no. 2, 379-400.

[28] S. L. Sun and Y. Wang, Reducing subspaces of certain analytic Toeplitz operators on the Bergman space, Northeast. Math. J., 14 (1998), no. 2, 147-158.

[29] S. Sun and D. Yu, Super-isometrically dilatable operators, Science in China, 32(12), 1989, 1447-1457.

[30] S. Sun and D. Yu, On unitary equivalence of multiplication operators on the Bergman space (II), Northeastern Math. J., 4(2) 1988, 169-179. 
[31] S. Sun, D. Zheng and C. Zhong, Classification of reducing subspaces of a class of multiplication operators on the Bergman space via the Hardy space of the bidisk, to appear in Canadian J. Math.

[32] S. Sun, D. Zheng and C. Zhong, Multiplication Operators on the Bergman Space and Weighted shifts, to appear in J. Operator Theory.

[33] J. Thomson, The commutant of a class of analytic Toeplitz operators, Amer. J. Math., 99(1977), 522-529.

[34] J. Thomson, The commutant of a class of analytic Toeplitz operators II, Indiana Univ. Math. J., 25(1976), 793-800.

[35] J. Walsh, On the location of the roots of the jacobian of two binary forms, and of the derivative of a rational function, Trans. Amer. Math. Soc., 19(1918), 291-298.

[36] J. Walsh, The location of critical points, Amer. Mat. Soc. Colloquium Publications, Vol 34, 1950.

[37] R. Yang, Operator theory in the Hardy space over the bidisk II, Integral Equations Operator Theory, 42 (2002), 99-124.

[38] R. Yang, Operator theory in the Hardy space over the bidisk III, J. Funct. Anal., 186 (2001), 521-545.

[39] R. Yang, The core operator and congruent submodules, J. Funct. Anal., 228 (2005), 469-489.

[40] K. Zhu, Reducing subspaces for a class of multiplication operators, J. London Math. Soc., (2) 62(2000), $553-568$.

[41] K. Zhu, Irreducible multiplication operators on spaces of analytic functions, J. Operator Theory, 51 (2004), no. $2,377-385$.

Kunyu Guo

Department of Mathematics

FUdAN UNIVERSITY

Shanghai, P. R. China

DechaO Zheng

DePARTMENT OF Mathematics

VANDERBILT UNIVERSITY

NAShVILle, TN 37240 USA

kyguo@fudan.edu.cn

dechao.zheng@vanderbilt.edu
Shunhua Sun

INSTITUTE OF MATHEMATICS

JiAXING UNIVERSITY

Jiaxing, Zhejiang, 314001, P. R. China

ChANGYONG ZHONG

Department of Mathematics \& Statistics

Georgia State University

Atlanta, Georgia 30303, USA

shsun@mail.zjxu.edu.cn matcyz@langate.gsu.edu 